Documentation et bibliothèques

DOCUMENTATION BIBLIOTHËQUES

\title{
La bibliothèque publique et le milieu municipal
}

Partie 2. Perception et support financier

The public library and the municipal environment

Part 2. Opinions and financial support

\section{La biblioteca pública y el medio municipal}

Parte 2. Percepción y ayuda financiera

\section{Diane Mittermeyer}

Volume 37, numéro 1, janvier-mars 1991

URI : https://id.erudit.org/iderudit/1028408ar

DOI : https://doi.org/10.7202/1028408ar

Aller au sommaire du numéro

\section{Éditeur(s)}

Association pour l'avancement des sciences et des techniques de la documentation (ASTED)

\section{ISSN}

0315-2340 (imprimé)

2291-8949 (numérique)

Découvrir la revue

\section{Citer cet article}

Mittermeyer, D. (1991). La bibliothèque publique et le milieu municipal : partie 2. Perception et support financier. Documentation et bibliothèques, 37(1), 5-21. https://doi.org/10.7202/1028408ar
Résumé de l'article

L'auteure établit un profil des perceptions de l'administration municipale face à la bibliothèque publique à partir de l'évaluation du degré de concordance entre les perceptions de chacun des groupes de répondants à une enquête réalisée auprès de 35 municipalités du Québec.

La place de la bibliothèque au sein des dépenses municipales du secteur Loisirs et Culture est étudiée et l'influence des perceptions de l'administration municipale sur le support financier accordé à la bibliothèque est discutée.
Tous droits réservés ( $)$ Association pour l'avancement des sciences et des techniques de la documentation (ASTED), 1991
Ce document est protégé par la loi sur le droit d'auteur. L'utilisation des services d'Érudit (y compris la reproduction) est assujettie à sa politique d'utilisation que vous pouvez consulter en ligne.

https://apropos.erudit.org/fr/usagers/politique-dutilisation/ 


\title{
La bibliothèque publique et le milieu municipal ${ }^{*}$ :
}

\author{
Partie 2: Perception et support financier
}

\author{
Diane Mittermeyer \\ Graduate School of Library and Information Studies \\ Université McGill \\ Montréal
}

L'auteure établit un profil des perceptions de l'administration municipale face à la bibliothèque publique à partir de l'évaluation du degré de concordance entre les perceptions de chacun des groupes de répondants à une enquête réalisée auprès de 35 municipalités du Québec.

La place de la bibliothèque au sein des dépenses municipales du secteur Loisirs et Culture est étudiée et l'influence des perceptions de l'administration municipale sur le support financier accordé à la bibliothèque est̆ discutée.

\section{The public library and the municipal environment - Part II: Opinions and financial support}

A group of 35 Québec municipalities was surveyed to determine their opinions regarding the public library. The author establishes a profile of the various opinions that municipal governments have towards the public library.

The portion of library-related expenses in the "recreation and culture" category of municipal budgets is analysed and the opinions of city administrators regarding the financial support of the library are discussed.

\section{La biblioteca pública y el medio municipal - Parte 2: Percepción y ayuda financiera}

La autora establece un perfil de las percepciones de la administración municipal frente a la biblioteca pública a partir de la evaluación del grado de concordancia entre las percepciones de cada grupo de respondientes a una encuesta realizada con 35 municipalidades de la provincia de Quebec.

Se estudia la importancia de la biblioteca en cuanto a los gastos municipales dentro del sector Ocio y cultura y se discute de la influencia de las percepciones de la administración municipal hacia la ayuda financiera otorgada a la biblioteca.
Dans un premier article ${ }^{1}$, nous avons présenté les résultats d'une enquête réalisée auprès de 35 municipalités du Québec et portant sur la perception de l'administration municipale face à la bibliothèque publique. Dans cette enquête, I'administration municipale incluait les élus municipaux, les cadres supérieurs (directeurs généraux et superviseurs hiérarchiques) et les responsables de bibliothèques. La perception fut évaluée selon l'importance attribuée à un nombre déterminé de facteurs caractérisant une bibliothèque publique. Bien que les résultats aient démontré que peu de ces facteurs furent perçus comme essentiels par la majorité des groupes de répondants, un grand nombre de facteurs furent cependant jugés importants.

Ce deuxième article fait d'abord le point sur les perceptions de l'administration municipale envers la bibliothèque publique et tente d'établir un profil de ces perceptions à partir de l'évaluation du degré de concordance entre les perceptions de chaque groupe. Puis, en ce qui a trait au support financier, nous analysons la place que la bibliothèque occupe dans la ventilation des dépenses municipales du secteur Loisirs et Culture. En dernier lieu, nous nous interrogeons sur la possibilité d'établir certains liens entre le profil des perceptions de l'administration municipale et le support financier accordé à la bibliothèque.

\section{Des perceptions qui diffèrent}

Pour évaluer l'importance accordée à certains facteurs caractérisant une bibliothèque publique, cinq choix possibles de réponses étaient offerts aux répondants: Essentiel, Important, Secondaire, Sans Importance, Sans Opinion. Une première analyse résume ce que majoritairement chaque groupe perçoit comme facteurs "essentiels et/ou importants". Bien qu'un bon nombre de ces facteurs soient effectivement perçus comme " essentiels et/ou importants" par la majorité des répondants, dans l'article précédent, nous suggérons "...une lecture des résultats à partir d'une majorité des $2 / 3$ ou $66 \%{ }^{2}$. Afin de dégager un consensus valable, à notre avis une lecture plus sévère des résultats s'impose. Par contre, comme

\footnotetext{
* Cette étude a été subventionnéé par l'Association pour I'avancement des sciences et des techniques de la documentation (ASTED), le ministère des Affaires culturelles et I'Union des municipalités du Québec.
}

1. Diane Mittermeyer, "La bibliothèque publique et le milieu municipal - Partie 1: La perception de l'administration municipale" Documentation et bibliothèques, vol.35, no 4 (octobre-décembre 1989), 145-161. (Voir Note à la fin du présent article)

2. Ibid., 151 
Tableau 1

Perception de I'administration municipale: ESSENTIEL et/ou IMPORTANT (majorité des $2 / 3$ pour chacun des groupes*)

Formes de documents :

Genres de livres:

Genres d'oeuvres musicales:

Équipements à la disposition des usagers :

Genres de services:

Genres d'activités d'animation:

Activités des élus:
1) livres, 2) revues, 3) journaux.

4) encyclopédies/dictionnaires, 5) best-sellers, 6) biographies, 7) oeuvres classiques, 8) documentaires, 9) livres pratiques, 10) bandes dessinées.

11) chansons enfantines, 12) folklore.

13) tables de travail avec chaises, 14) coin lecture pour les enfants,

15) fauteuils confortables pour la lecture de détente, 16) photocopieurs.

17) consultation sur place, 18) prêts de documents, 19) réservation de documents, 20) prêts entre bibliothèques, 21) aide aux lecteurs, 22) suggestions et demandes spéciales, 23) référence.

24) heure du conte, 25) rencontres avec des auteurs, 26) ateliers sur l'apprentissage de la lecture.

27) discuter de la bibliothèque avec le responsable en dehors des réunions du conseil, 28) visiter la bibliothèque régulièrement, 29) faire la promotion des services offerts par la bibliothèque auprès des groupes et organismes de la communauté, 30) participer activement à l'élaboration des buts et objectifs de la bibliothèque, 31) planifier un programme de développement à moyen et long termes des services offerts par la bibliothèque, 32) visiter $d^{\prime}$ autres bibliothèques publiques, 33) discuter du service de la bibliothèque avec des élus d'autres municipalités.

* Extrait de Diane Mittermeyer, "La bibliothèque publique et le milieu municipal - Partie $1: \ldots . ., 151$.

le montre le tableau 1, même avec une lecture plus sévère, près de la moitié des facteurs (33 sur 67) sont perçus comme "essentiels et/ou importants ".

Bien qu'une première analyse descriptive tende à démontrer un certain consensus, il est apparu important de vérifier si les perceptions différaient de façon significative entre les groupes. Le tableau 2 et le tableau 3 présentent les résultats du test statistique KruskalWallis. Ce test permet d'identifier les variables pour lesquelles des différences de perception statistiquement significatives existent entre les groupes ${ }^{3}$.

Les résultats de ces tests montrent que pour 21 variables (tableau 2) il n'y a pas de différence de perception statistiquement significative entre les quatre groupes, soit le groupe des élus municipaux, celui des directeurs généraux, celui des directeurs hiérarchiques, et finalement le groupe des responsables de bibliothèques. Par contre le tableau 3 présente 46 varia- bles pour lesquelles les perceptions diffèrent de façon significative.

\section{Profil des perceptions par regroupements}

À partir des résultats présentés aux tableaux 1, 2 et 3, des regroupements de variables sont possibles permettant ainsi d'établir un certain profil de perceptions. Le premier regroupement porte sur l'identification des variables communes aux tableaux 1 et 2 . Ces onze variables sont:

1) livres;

2) oeuvres classiques ;

3) chansons enfantines;

4) folklore ;

5) consultation sur place ;

6) ateliers sur l'apprentissage de la lecture;

7) faire la promotion des services offerts par la bibliothèque auprès des groupes et organismes de la communauté ;
8) participer activement à l'élaboration des buts et objectifs de la bibliothèque ;

9) planifier un programme de développement à moyen et long termes des services offerts par la bibliothèque ;

10) visiter d'autres bibliothèques publiques ;

11) discuter du service de la bibliothèque avec des élus $d$ 'autres municipalités.

Ces variables présentent un message assez clair de la perception des quatre groupes: au moins les $2 / 3$ des répondants de chaque groupe les perçoivent comme " essentielles et/ou importantes" (tableau 1), et il n'y a pas de différence de perception significative entre les groupes (tableau 2).

3. Sidney Seigel and N. John Castellan, Jr. Nonparametric Statistics for the Behavioral Sciences, 2nd ed., New York, McGraw-Hill, 1988, p. 206-212. 
Genres de livres :

Genres d'oeuvres musicales: Équipements à la disposition
des usagers:

\section{Genres de services:}

Genres d'activités d'animation :

\section{Activités des élus :}

1) livres**

2) disques

3) vidéo-cassettes

4) logiciels d'ordinateur

5) oeuvres classiques**

6) chansons enfantines**

7) folklore**

8) appareils pour écouter disques, cassettes ou disques compacts

9) appareils pour visionner des vidéo-cassettes

10) ordinateurs personnels

11) consultation sur place co $^{*}$

12) ateliers sur l'apprentissage de la lecture**

13) rencontres avec des philatélistes

14) organiser, sur une base annuelle, une rencontre avec les citoyens pour discuter des services offerts par la bibliothèque;

15) assister aux activités d'animation organisées par la bibliothèque ;

16) assister à des conférences, colloques ou congrès, portant sur les bibliothèques publiques ;

17) faire la promotion des services offerts par la bibliothèque auprès des groupes et organismes de la communauté** ;

18) participer activement à l'élaboration des buts et objectifs de la bibliothèque ${ }^{* *}$;

19) planifier un programme de développement à moyen et long termes des services offerts par la bibliothèque ${ }^{* *}$;

20) visiter $d^{\prime}$ autres bibliothèques publiques ${ }^{* *}$;

21) discuter du service de la bibliothèque avec des élus d'autres municipalités**.
Niveau de signification*

.2460

.5070

.0975

.3763

.1686

.0600

.1776

.6488

.1919

.1884

.0539

.4003

.3734

.2812

.0767

.2143

.2493

.2857

.4850

.0850

.4909

* Résultats du test statistique Kruskal-Wallis, significatif à .05.

* Ces variables ont également été perçues comme "Essentiel et/ou Important " par une majorité des $2 / 3$ des répondants pour chacun des groupes.

Ce premier regroupement nous permet de constater que bien peu de facteurs sont perçus de la même manière, ou au même niveau, par I'ensemble des répondants en ce qui a trait aux éléments essentiels ou du moins importants caractérisant une bibliothèque publique. En fait, au niveau des Formes de documents (livres), des Genres de livres (oeuvres classiques), des Genres d'oeuvres musicales (chansons enfantines et folklore), ainsi que des Genres de services (consultation sur place); ce profil des perceptions nous apparaît très limité et conservateur. Il est à noter que dans la catégorie Équipements à la disposition des usagers, aucun équipement particulier n'a été retenu, et en ce qui concerne les Genres d'activités d'animation, seuls les Ateliers sur l'apprentissage de la lecture ont retenu l'attention. Sur ce dernier point on peut noter une certaine constance puisque les seuls documents retenus sont les livres.
D'autre part, en ce qui a trait aux cinq Activités des élus, il semble bien que tous les groupes favorisent un plus grand engagement des élus dans le "dossier bibliothèque ".

Le deuxième regroupement consiste à identifier les dix variables qui restent au tableau 2. De nouveau, le message est assez clair. Premièrement, il n'y a pas de différence de perception significative entre les groupes et, deuxièmement, ces varia- 
bles ne se retrouvent pas au tableau 1 , donc, elles ne sont pas perçues comme "essentielles et/ou importantes" par les $2 / 3$ des répondants de chacun des groupes. Ces variables sont:

1) disques;

2) vidéo-cassettes;

3) logiciels d'ordinateur;

4) appareils pour écouter disques, cassettes ou disques compacts;

5) appareils pour visionner des vidéocassettes ;

6) ordinateurs personnels ;

7) rencontres avec des philatélistes;

8) organiser, sur une base annuelle, une rencontre avec les citoyens pour discuter des services offerts par la bibliothèque ;

9) assister aux activités d'animation organisées par la bibliothèque;

10) assister à des conférences, colloques ou congrès, portant sur les bibliothèques publiques.

Une constance assez marquée se. dégage de ce deuxième regroupement. Les Formes de documents (disques, vidéo-cassettes, logiciels d'ordinateur) et Équipements (les appareils nécessaires à l'utilisation de ces documents) vont de pair. Par contre, si pour des raisons de qualité technique nous avons de plus en plus tendance à abandonner le disque 33 tours, les autres formes de documents de cette liste ainsi que les appareils nécessaires à leur utilisation font désormais partie du quotidien de beaucoup d'usagers de bibliothèque, qu'on le perçoive ainsi ou non. Ces usagers pourraient sans doute s'attendre à retrouver ces éléments de leur vie quotidienne dans leur bibliothèque.

Par contre les trois Activités des élus de la présente liste confirment les activités de la liste précédente. Par exemple, point n'est besoin d'assister aux activités d'animation offertes par la bibliothèque, puisque la seule activité que l'on perçoit comme "essentielle et/ou importante" concerne l'organisation d'ateliers sur l'apprentissage de la lecture. Par contre, le fait que l'on perçoive comme important que les élus participent, planifient, et fassent la promotion de la bibliothèque, mais que l'on perçoive comme beaucoup moins important que de telles activités s'accomplissent dans un contexte de consultation des citoyens, reflète à notre avis une situation assez répandue dans notre société québécoise. Situation que Baccigalupo décrit en termes de démocratie représentative, et qui consiste à assurer "... une représentation équitable des citoyens aux divers postes électifs liés à l'exercice du pouvoir local ", mais qui "...n'a pour effet que d'engendrer un style de démocratie très formel $" 4$.

D'autre part, en ce qui concerne la formation des élus par le biais de conférences, colloques ou congrès portant sur les bibliothèques publiques, il faut noter qu'au Québec, la tenue de ces conférences, colloques ou congrès est une denrée plutôt rare. Bien que I'Association des directeurs de bibliothèques publiques du Québec (ADIBIPUQ), consciente de l'importance de la formation de ses membres, organise parfois un Forum, son marché cible n'est pas, du moins pour le moment, celui des élus municipaux. Pour certains autres organismes, comme I'Union des municipalités du Québec, une analyse des calendriers des sessions de formation ne montre aucune activité de formation des élus dans le domaine des bibliothèques, bien que depuis 1984 la formation des élus soit une priorité 5 .

Le message des deux derniers regroupements est moins clair et, à vrai dire, inquiète quelque peu. Le troisième regroupement porte sur l'identification des variables communes aux tableaux 1 et 3 . Celles-ci sont au nombre de 22 et représentent ce que les $2 / 3$ de chaque groupe perçoivent comme "essentiel et/ou important", mais dont les niveaux d'importance entre les groupes diffèrent de manière significative. Ces variables sont:
1) revues
2) journaux
3) encyclopédies/dictionnaires
4) best-sellers
5) biographies
6) documentaires
7) livres pratiques
8) bandes dessinées
9) tables de travail avec chaises
10) coin lecture pour les enfants

11) fauteuils confortables pour la lecture de détente

12) photocopieurs

13) prêts de documents

14) réservation de documents

15) prêts entre bibliothèques

16) aide aux lecteurs

17) suggestions et demandes spéciales

18) référence

19) heure du conte

20) rencontres avec des auteurs

21) discuter de la bibliothèque avec le responsable en dehors des réunions du conseil

22) visiter la bibliothèque régulièrement:

Lorsque I'on met en parallèle ce troisième regroupement avec les deux précédents, une constatation s'impose. La perception de l'administration municipale envers la bibliothèque publique, telle qu'exprimée par les quatre groupes participant à cette enquête, affiche, à notre avis, une constance troublante pour l'avenir des bibliothèques publiques au Québec. Certains membres, ou peut-être même certains groupes, de l'administration municipale ont vraisemblablement une perception limitée du potentiel d'une bibliothèque pub'lique moderne. Le quatrième et dernier regroupement consiste en une. liste de 24 variables (tableau 3) pour lesquelles les perceptions diffèrent de façon significative entre les quatre groupes et qui n'apparaissent pas au tableau 1. Ces variables ne sont donc pas perçues comme "essentielles et/ou importantes " par les $2 / 3$ des répondants de chacun des groupes. Ces variables sont :

1). cassettes

2) disques compacts

3) oeuvres d'art

4) jeux éducatifs

5) rock

6) jazz

7) musique classique

4. Alain Baccigalupo, Les administrations municipales québécoises des origines à nos jours, Tome II: L'environnement, Montréal, Agence d'ARC, 1984, p.1006.

5. «Dossier formation - La formation : au service des élus", Urba, vol. 11, no 10 (décembre 1990 - janvier 1991), 9. 
Tableau 3

Variables pour lesquelles les perceptions diffèrent de façon significative entre les quatre groupes

Formes de documents:
1) cassettes
2) disques compacts
3) revues**
4) journaux**
5) oeuvres d'art
6) jeux éducatifs

Niveau de signification*

Genres de livres:
7) encyclopédies/dictionnaires**
8) best-sellers**
9) biographies**
10) documentaires**
11) livres pratiques**
12) bandes dessinées**

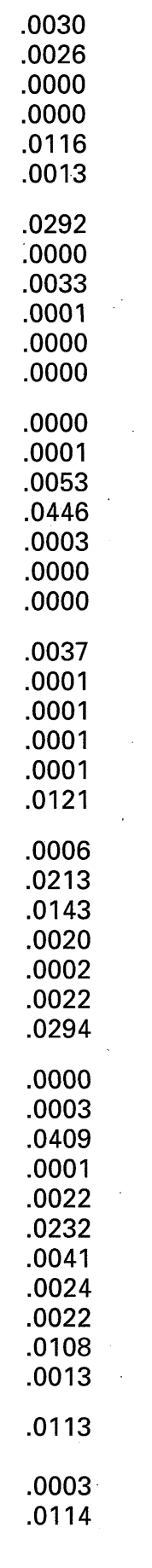

Équipements à la

disposition des usagers :

Genres de services:

\section{Genres d'activités d'animation:}

Activités des élus:
13) rock

14) jazz

15) musique classique

16) opéra

17) country

18) heavy metal

19) chansons populaires

20) tables de travail avec chaises**

21) coin lecture pour les enfants**

22) fauteuils confortables pour la lecture de détente**

23) photocopieurs**

24) salles d'expositions et de conférences

25) laboratoire de langues

26) prêts de documents**

27) réservation de documents**

28) prêts entre bibliothèques**

29) aide aux lecteurs**

30) suggestions et demandes spéciales**

31) référence ${ }^{* *}$

32) référence téléphonique

33) heure du conte ${ }^{* *}$

34) rencontres avec des auteurs**

35) rencontres avec des experts dans le domaine de la nutrition

36) atelier sur la tenue du budget familial

37) rencontres avec des experts dans le domaine de la santé

38) rencontres avec des experts dans le domaine de l'actualité

39) expositions de peinture

40) cours de langues

41) rencontres avec des experts en voyage

42) ateliers sur le jardinage

43) rencontres avec des experts dans le domaine juridique

44) discuter de la bibliothèque avec le responsable en dehors des réunions du conseil**

45) visiter la bibliothèque régulièrement**

46) consulter les représentants du ministère des Affaires culturelles responsables du développement des bibliothèques publiques

* Résultats du test statistique Kruskal-Wallis, significatif à .05.

** Ces variables ont également été perçues comme "Essentiel et/ou Important " par une majorité des 2/3 des répondants pour chacun des groupes. 

8) opéra
9) country
10) heavy metal
11) chansons populaires
12) salles d'exposition et de confé- rences
13) laboratoire de langues
14) référence téléphonique
15) rencontres avec des experts dans le domaine de la nutrition
16) ateliers sur la tenue du budget familial

17) rencontres avec des experts dans le domaine de la santé

18) rencontres avec des experts dans le domaine de l'actualité

19) expositions de peinture

20) cours de langue

21) rencontres avec des experts en voyage

22) ateliers sur le jardinage

23) rencontres avec des experts dans le domaine juridique

24) consulter les représentants du ministère des Affaires culturelles responsables du développement des bibliothèques publiques.

Quand ils réfléchissent aux Formes de documents, il semble que les répondants considèrent que seul le document écrit (livres, revues, journaux) peut être perçu comme faisant partie intégrante d'une bibliothèque, et que la place des revues et journaux sur les rayons de la bibliothèque $n^{\prime}$ est pas nécessairement assurée. Même les Genres de livres suggèrent des perceptions variées quant à leur importance, sauf bien entendu les oeuvres classiques. Que l'importance des best-sellers ou des bandes dessinées suscite des différences de perception, ceci peut refléter une vision de la bibliothèque publique davantage orientée vers la diffusiỏn des connaissances et de l'information. Mais le fait que les encyclopédies, les dictionnaires, les biographies et les documentaires suscitent également une différence de perception entre les quatre groupes, devrait inquiéter les professionnels de l'information. Même si cette différence n'est que de l'ordre de " important " à " essentiel ".

Les Genres d'oeuvres musicales se méritent à peu près le même sort que les Genres d'activités d'animation. En effet, une majorité des répondants (les deux-tiers) ne perçoivent pas les oeuvres musicales et les activités d'animation comme des outils nécessaires à la diffusion des connaissances et de l'information au sein d'une bibliothèque publique. Dans le domaine des Equipements mis à la disposition des usagers, la consultation sur place étant le seul "Genre de services " pour lequel il n'y a pas de différence de perception, il convient que les tables de travail et les fauteuils confortables fassent partie du groupe " essentiel et/ou important " bien que, là encore, leur importance ne fasse pas I'unanimité. D'autre part, les différences de perception des Genres de services surprennent tout autant que celles constatées pour les Genres de livres.

En dernier lieu, que dire des activités des élus que l'on retrouve au troisième et au quatrième regroupements ! Encore une fois, force nous est de souligner une certaine constance dans l'esprit de nos administrateurs municipaux, ou du moins chez certains groupes. Il semble bien que les élus municipaux aient la responsabilité de s'engager activement, de planifier et de promouvoir les intérêts de la bibliothèque, mais que ce travail doive se faire, somme toute, en vase clos.

En conclusion de cette première partie, une question se pose: "La bibliothèque publique serait-elle une institution méconnue de nos administrateurs municipaux québécois?" Selon le tableau 1, nous serions tentés de répondre "Non ». Mais si l'on tient compte des tableaux 2 et 3 , peut-être gagnerions-nous à faire connaître davantage cette institution. Encöre faut-il qu'une connaissance plus approfondie puisse contribuer à aplanir certaines différences de perception entre élus, directeurs généraux, directeurs hiérarchiques, et directeurs de bibliothèques municipales. Mais si on en croit certains commentaires écrits à la fin du questionnaire d'enquête de cette étude, le travail pourrait être ardu. À titre d'exemple, un élu écrit:

Je ne suis pas certain que la hiérarchie permet au directeur de la bibliothèque de discuter avec les élus municipaux.

La bibliothèque devrait dépendre directement du directeur général et du conseil et non du service des loisirs, où elle a très peu d'affinités.
Un responsable de bibliothèque écrit:

Je peux vous dire qu'il n'y a aucun problème de discussion avec nos élus municipaux, mais la discussion se fait avec mon patron.

Un directeur général fait le commentaire suivant:

Pas question pour moi de recommander de faire de la bibliothèque un service relevant directement $d u$ D.G. Elle est très bien placée aux Services...

Nous tenons à préciser que ces commentaires proviennent de trois municipalités différentes et qu'en ce qui concerne le troisième commentaire, le service auquel appartient la bibliothèque n'a pas été identifié afin de conserver l'anonymat du répondant et de sa municipalité.

\section{Le support financier}

Comme le souligne le Rapport de la Commission d'étude sur les bibliothèques publiques du Québec, "...les municipalités du Québec contribuent pour $69,5 \%$ du financement des bibliothèques publiques..." 6 . Un rapport détaillé de ce support financier est. d'ailleurs publié chaque année par le ministère des Affaires culturelles dans un document intitulé Statistiques 19--: Bibliothèques publiques du Québec. Ce document permet de suivre, année après année, l'évolution des revenus et des dépenses des bibliothèques publiques. Les données des'Statistiques du ministère des Affaires culturelles ne permettent pas cependant d'analyser la participation des municipalités au financement de leur bibliothèque dans le contexte de certaines autres dépenses municipales à caractère culturel ou de loisirs. À notre avis, une telle analyse s'avère utile et nécessaire car elle permet, dans une certaine mesure, une évaluation de l'effort financier municipal envers la bibliothèque par rapport à l'effort financier consenti au secteur Loisirs et Culture.

6. Québec. Commission d'étude sur les bibliothèques publiques du Québec, Les bibliothèques publiques - une responsabilité à partager, rapport, Québec, La Commission, 1987, p. 53. 
Comme nous le mentionnions dans un article précédent:

Au niveau du secteur des loisirs, il semble, au Québec, que les municipalités aient d'abord favorisé les sports en supportant financièrement et en administrant certains services "sportifs" tels le centre sportif, la piscine, les patinoires, les terrains de jeux. L'ouverture des municipalités aux services "culturels", tels la bibliothèque, le patrimoine, le centre ou la maison de la culture, semble être beaucoup plus récente ${ }^{7}$.

Par contre, selon Maud LefebvreRoux, présidente de l'Association des directeurs de bibliothèques publiques du Québec, les municipalités ont définitivement amorcé un virage culturel:

Les temps changent et les besoins se transforment. La priorité est passée des équipements sportifs aux équipements culturels, la vague des arénas est dépassée, les Québécois aspirent désormais à des activités culturelles dans leur milieu de vie. Les municipalités investissent donc de plus en plus de ressources financières et humaines dans le développement culturel et cela se traduit par des restructurations administratives importantes ${ }^{8}$.

Devant de tels changements, il est donc à propos de vérifier dans quelle mesure l'administration municipale apporte son support financier au "dossier bibliothèque".

\section{Méthodologie}

Puisque les données du ministère des Affaires culturelles ne nous permettaient pas d'effectuer l'analyse désirée, I'utilisation d'une autre source de données financières municipales valable et reconnue était requise. II fut décidé d'utiliser les données du rapport financier municipal. Selon Lise Caron, comptable agréée et directrice générale de l'administration financière au ministère des Affaires municipales, le rapport financier constitue:

...un outil de références indispensable à un grand nombre d'utilisateurs que ce soit les municipalités, les citoyens, les gouvernements ou les institutions financières, ...9
Aussi :

À l'étape de la préparation du rapport financier, les municipalités font le point sur leur situation financière, analysent leurs résultats et rendent des comptes, notamment, à leurs citoyens. Le rapport financier leur permet de traduire, en termes monétaires, les activités effectuées, les gestes posés et les décisions prises tout au cours de l'année. Le rapport financier constitue donc un instrument privilégié de communication de l'information fináncière ${ }^{10}$.

II faut se rappeler que le rapport financier de la municipalité est en quelque sorte l'image fidèle et officielle de la municipalité. La municipalité est responsable des renseignements qui y sont présentés et doit donc se préoccuper de la qualité de l'information qui $y$ est contenue ${ }^{11}$.

De plus, I'utilisation du rapport financier municipal permet de mettre en perspective le support financier accordé à la bibliothèque par rapport à celui qui est apporté à un ensemble similaire de services. En effet, le rapport financier regroupe par rubriques certains postes budgétaires. Par exemple, sous la rubrique Hygiène du Milieu, on retrouve la purification et le traitement de l'eau, le réseau de distribution de l'eau, le réseau d'égouts, ainsi que l'enlèvement et la destruction des ordures. Sous la rubrique Urbanisme et Mise en Valeur du Territoire sont regroupés le zonage, la promotion et le développement industriels, la rénovation urbaine et la restauration, ainsi que le logement.

Si un tel regroupement suggère un certain lien entre ces postes, une analyse du budget sous la rubrique Loisirs et Culture peut s'avérer fort intéressante compte tenu du fait que le rapport financier traduit "...en termes monétaires, les activités effectuées, les gestes posés et les décisions prises...".

Cependant, il va sans dire que l'analyse comparative de données financières municipales commande la prudence. Le Rapport de la Commission d'étude sur les municipalités soutient que:

... La description des fonctions municipales à partir des postes budgétaires des municipalités, tels que définis dans le Manuel de normalisation, bien qu'utile pour donner un aperçu des services fournis par les municipalités, renseigne cependant peu sur l'ampleur de ces activités. L'importance absolue et relative des différents services municipaux ressort toutefois nettement lorsque l'on s'attarde aux dépenses effectuées par les municipalités du Québec ${ }^{12}$.

Ce même rapport fait cependant trois mises en garde aux utilisateurs de ce genre de statistiques:

En premier lieu, l'imputation de certaines dépenses à un poste budgétaire spécifique donne souvent lieu à interprétation de la part des administrations municipales, rendant ainsi difficile la comparaison des données entre municipalités...

En second lieu, dépenses élevées n'est pas synonyme de quantité accrue. II ne faudrait pas croire qu'une municipalité qui dépense davantage pour une fonction spécifique offre nécessairement plus de services... En optant pour l'approche "dépenses", on se réfère bien plus aux coûts d'un service qu'à la quantité prodùite...

Enfin, s'il n'est pas adéquat de relier directement dépenses et niveau de services, il en est certes de même pour dépenses et qualité des services. L'ampleur des dépenses affectées à une fonction municipale ne doit aucunement être interprétée comme un guide de la qualité des services offerts ${ }^{13}$

Par contre, il est heureux que les commissaires ajoutent: "Ces réserves étant faites, on analysera maintenant

7. Diane Mittermeyer, «La bibliothèque publique et le milieu... "n, 148.

8. Maud Lefebvre-Roux, "Les directeurs de bibliothèques publiques face au virage culturel ", Défi, vol. 5, no 2 (août 1990), 6 .

9. Lise Caron, "Le rapport financier 1990 . consolider les acquis ", Municipalité, (janvier 1991), 9.

10. Ibid.

11. Ibid.

12. Rapport de la Commission d'étude sur les municipalités, Montréal, Union des municipalités du Québec, 1986, p. 84

13. Ibid., p. 84-85 
Tableau 4

Poste budgétaire occupant le premier rang au niveau des dépenses du secteur Loisirs et Culture

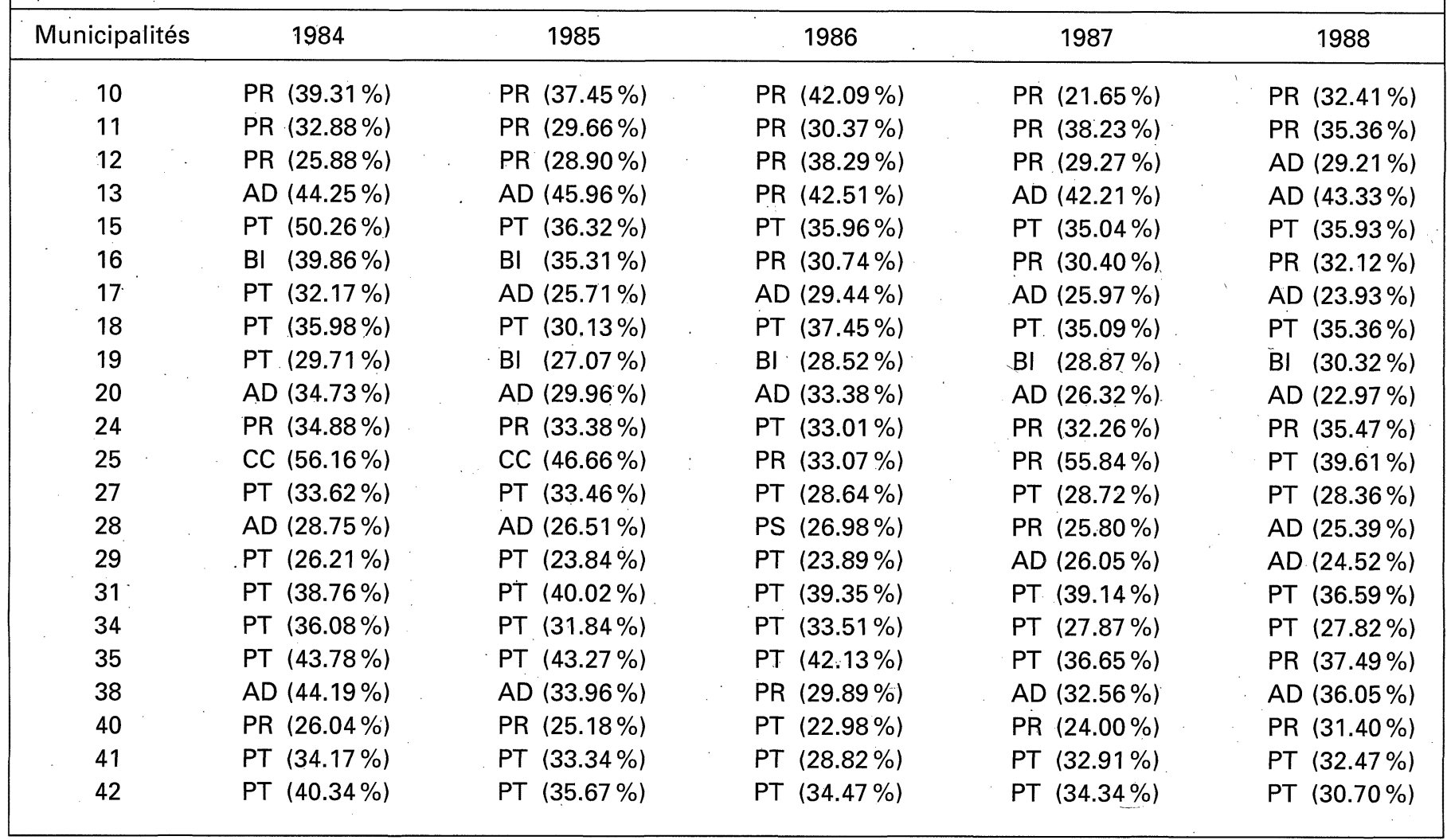

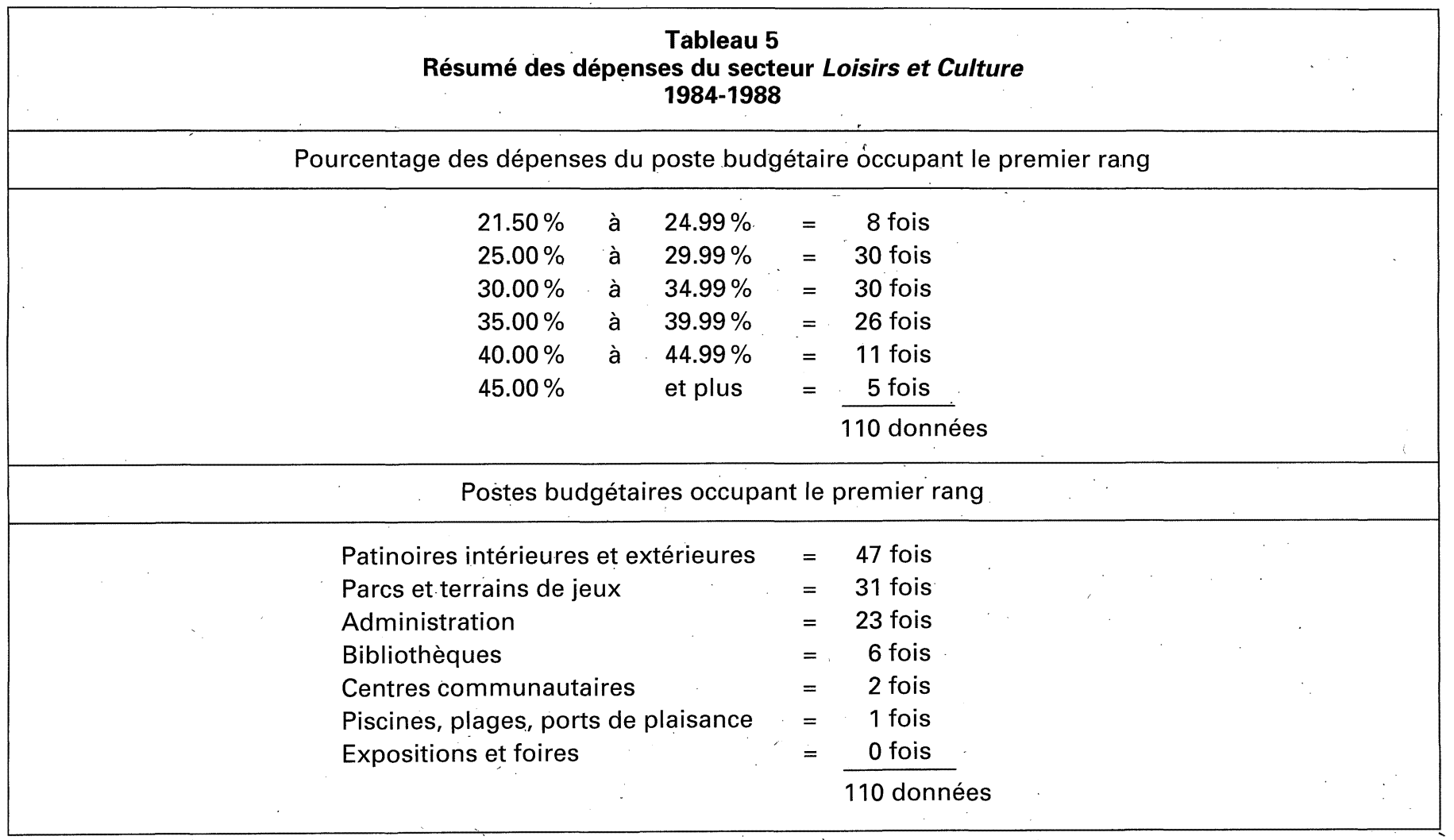


Tableau 6

Rang de la bibliothèque en $\%$ des dépenses du secteur Loisirs et Culture

\begin{tabular}{|rrrrrrr|}
\hline Municipalités & 1984 & 1985 & \multicolumn{1}{c}{1986} & 1987 & 1988 \\
\hline 10 & $12.85 \%(4)$ & $11.33 \%(5)$ & $12.44 \%(5)$ & $14.79 \%(4)$ & $14.30 \%(4)$ \\
11 & $8.65 \%(4)$ & $8.97 \%(4)$ & $7.44 \%(5)$ & $6.76 \%(5)$ & $6.87 \%(6)$ \\
12 & $25.24 \%(2)$ & $18.52 \%(3)$ & $16.29 \%(3)$ & $19.20 \%(3)$ & $21.02 \%(3)$ \\
13 & $14.96 \%(3)$ & $15.38 \%(3)$ & $13.19 \%(3)$ & $12.82 \%(4)$ & $12.81 \%(3)$ \\
15 & $18.88 \%(2)$ & $16.12 \%(3)$ & $18.16 \%(2)$ & $20.01 \%(2)$ & $18.68 \%(2)$ \\
16 & $39.86 \%(1)$ & $35.31 \%(1)$ & $30.06 \%(2)$ & $26.05 \%(2)$ & $19.26 \%(2)$ \\
17 & $15.84 \%(4)$ & $12.67 \%(4)$ & $13.30 \%(4)$ & $13.49 \%(4)$ & $13.55 \%(5)$ \\
18 & $9.43 \%(4)$ & $10.08 \%(5)$ & $12.92 \%(3)$ & $13.43 \%(4)$ & $12.88 \%(4)$ \\
19 & $27.14 \%(2)$ & $27.07 \%(1)$ & $28.52 \%(1)$ & $28.87 \%(1)$ & $30.32 \%(1)$ \\
20 & $12.03 \%(4)$ & $11.92 \%(5)$ & $12.38 \%(5)$ & $11.36 \%(5)$ & $15.21 \%(3)$ \\
24 & $9.28 \%(5)$ & $10.65 \%(4)$ & $11.43 \%(4)$ & $11.95 \%(4)$ & $10.97 \%(4)$ \\
25 & $7.38 \%(3)$ & $36.55 \%(2)$ & $23.61 \%(2)$ & $38.23 \%(2)$ & $18.21 \%(4)$ \\
27 & $16.62 \%(3)$ & $14.42 \%(4)$ & $13.34 \%(5)$ & $13.42 \%(5)$ & $14.96 \%(4)$ \\
28 & $19.47 \%(2)$ & $11.03 \%(6)$ & $9.07 \%(6)$ & $9.76 \%(6)$ & $9.90 \%(6)$ \\
29 & $18.06 \%(4)$ & $15.26 \%(4)$ & $11.91 \%(5)$ & $11.65 \%(5)$ & $13.33 \%(5)$ \\
31 & $9.39 \%(5)$ & $9.57 \%(5)$ & $9.15 \%(4)$ & $6.13 \%(5)$ & $9.21 \%(5)$ \\
34 & $11.43 \%(4)$ & $12.38 \%(4)$ & $12.81 \%(4)$ & $11.59 \%(4)$ & $11.41 \%(4)$ \\
35 & $7.37 \%(5)$ & $7.41 \%(4)$ & $7.61 \%(4)$ & $9.34 \%(4)$ & $9.14 \%(4)$ \\
38 & $4.29 \%(5)$ & $11.13 \%(3)$ & $21.25 \%(3)$ & $16.55 \%(3)$ & $14.09 \%(3)$ \\
40 & $11.90 \%(5)$ & $12.03 \%(4)$ & $13.99 \%(4)$ & $14.82 \%(4)$ & $14.05 \%(4)$ \\
41 & $15.67 \%(2)$ & $17.99 \%(2)$ & $17.01 \%(2)$ & $20.77 \%(3)$ & $25.40 \%(2)$ \\
42 & $16.35 \%(4)$ & $12.21 \%(4)$ & $12.71 \%(4)$ & $13.45 \%(4)$ & $13.51 \%(4)$ \\
& & & $(4)$ & $(4)$ & $(4)$ \\
\end{tabular}

les dépenses des municipalités du Québec "14. En somme, ce que Jacques Parizeau et ses collègues de la Commission nous disent, c'est que l'analyse par postes budgétaires des dépenses municipales fait nettement ressortir l'importance absolue et relative des différents services municipaux, mais qu'une analyse des dépenses n'est, en fait, qu'une analyse des coûts d'un service, rien de plus. Par contre si, comme le souligne Lise Caron, ces dépenses ou ces coûts sont la traduction des activités effectuées, des gestes posés et des décisions prises pendant l'année, alors l'analyse des données financières municipales de la présente étude ne vise qu'à faire ressortir l'importance "absolue et/ou relative" de certains services municipaux du secteur Loisirs et Culture.

De ce secteur, les sepi postes suivants furent retenus:

1) Administration (AD)

2) Centres communautaires (CC)

3) Patinoires intérieures et extérieures (PT)
4) Piscines, plages, ports de plaisance (PS)

5) Parcs et terrains de jeux (PR)

6) Expositions et foires (EX)

7) Bibliothèques (BI)

et les rapports financiers de 22 municipalités pour une période de cinq ans (1984 - 1988) furent analysés.

Les 22 municipalités de la présente analyse proviennent de l'enquête mentionnée précédemment ${ }^{15}$. Le critère de sélection consiste en un taux de réponse de $66 \%$ des élus de chacune des municipalités participantes ${ }^{16}$. Puisqu'en moyenne le nombre d'élus au conseil municipal varie entre 7 et 9 , il était important à notre avis de ne considérer du point de vue support financier que les municipalités où une majorité relativement importante d'élus avait manifesté son intérêt en participant à l'enquête sur les perceptions.

En dernier lieu nous aimerions préciser que toutes les données de cette analyse, couvrant les années 1984 à 1988 inclusivement, furent mises à notre disposition par le ministère des Affaires municipales du Québec. De plus, si, comme le mentionne Lise Caron, le rapport financier municipal a connu de grands changements en 1989 17, de 1984 à 1988 aucun changement majeur ne fut effectué.

\section{Les résultats}

La première analyse consiste à évaluer, pour chaque municipalité, le

14. Ibid., p. 85.

15. Pour une présentation détaillée du choix de ces municipalités, voir: Diane Mittermeyer, "La bibliothèque publique et le milieu..." , 149.

16. Ibid., 150 (Tableau 2 - Taux de réponse par municipalité).

17. Nous tenons à remercier le ministère des Affaires municipales pour la promptitude et la diligence avec lesquelles il a répondu à notre demande. 


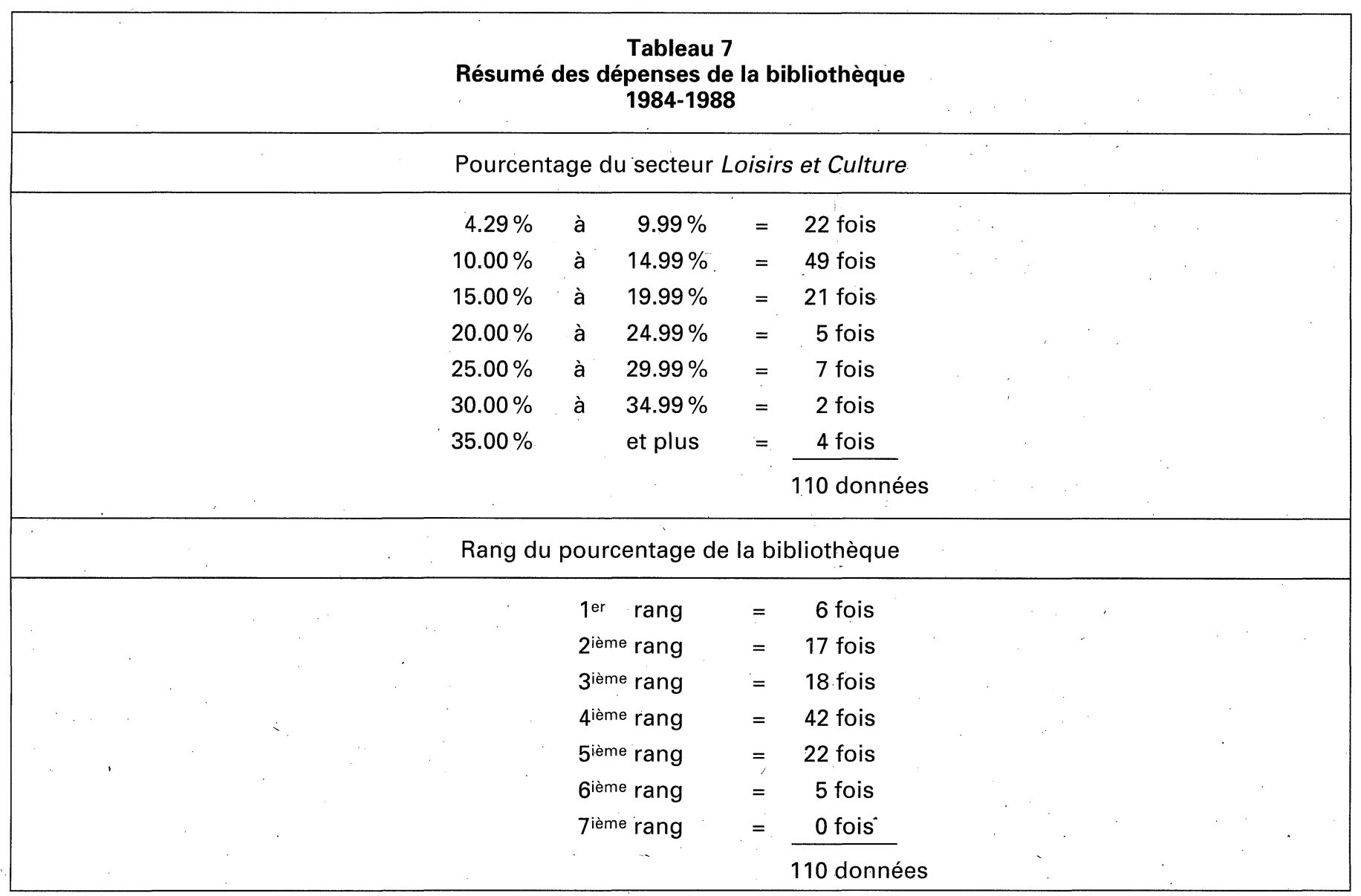

poste budgétaire occupant le premier rang au niveau des dépenses du secteur Loisirs et Culture à partir du pourcentage des dépenses consenties à ce poste par rapport à I'ensemble des dépenses de ce secteur. Les tableaux 4 et 5 présentent ces résultats.

II ne s'agit aucunement de comparer les municipalités entre elles, mais plutôt de présenter, pour une période de cinq ans, un profil des différents postes budgétaires qui occupèrent le premier rang. Comme on peut le constater, la priorité semble aller aux patinoires (PT) intérieures (arénas !) et extérjeures ainsi qu'aux parcs (PR) et aux terrains de jeux; et le plus souvent ce premier poste englobe entre $25 \%$ et $40 \%$ du budget de dépenses du secteur Loisirs et Culture.

Puisque les bibliothèques n'occupent que très rarement le premier rang, la deuxième analyse présente une évaluation de la place de la bibliothèque parmi les dépenses du secteur Loisirs et Culture. (tableaux 6 et 7)
Dans la plupart des municipalités, le budget de la bibliothèque occupe le plus souvent le quatrième ou le cinquième rang dans l'ensemble du budget du secteur Loisirs et Culture. Et dans une très grande proportion ce pourcentage est inférieur à $15 \%$. Afin de faciliter la comparaison de ces deux postes budgétaires (premier rang et bibliothèque) par municipalité, le tableau 8 présente une vue d'ensemble pour la période à l'étude.

Devant de tels résultats; il est permis de conclure qu'entre 1984 et 1988 les gestes posés et les décisions prises, du moins ceux traduits en termes monétaires; au niveau des loisirs et de la culture ne semblent pas avoir beaucoup favorisé les bibliothèques. Tenant compte de la première mise en garde du rapport de la Commission d'étude sur les municipalités, nous tenons à répéter que cette analyse ne vise pas une comparaison entre municipalités, mais établit plutôt une comparaison à l'interne, entre deux postes d'un même secteur. Si, de plus, des dépenses élevées ne sont pas synonyme de quantité accrue ni de qualité des services offerts, nous sommes en droit de nous demander sur quel(s) critère(s) les administrateurs municipaux décident d'allouer $30 \%$ ou même $35 \%$ du budget d'un secteur aussi vaste que les loisirs et la culture aux patinoires (arénas !) ou aux parcs et terrains de jeux. Aussi, force nous est de constater que si "la priorité est passée des équipements sportifs aux équipements culturèls,..." ", ce phénomène est sûrement très récent.

Puisque dans une forte proportion, ce sont les patinoires (PT) et les parcs (PR) qui occupent le premier rang, mais que l'intérêt premier de cette analyse porte sur les bibliothèques (BI), une analyse de la croissance ou de la décroissance de ces postes budgétaires pour la période 1984 1988 fut réalisée. Le tableau 9 présente les résultats de ces changements.

Ainsi, nous constatons que les Patinoires ont connu 62 fois une croissance $(+)$, et 19 fois une décroissance $(-)$, tenant compte des 7 fois où 


\begin{tabular}{|c|c|c|c|c|c|}
\hline \multicolumn{6}{|c|}{$\begin{array}{l}\text { Tableau } 8 \\
\text { Comparaison entre le \% des dépenses de la bibliothèque et le \% des dépenses du poste } \\
\text { occupant le premier rang du secteur Loisirs et Culture }\end{array}$} \\
\hline Municipalités & 1984 & 1985 & 1986 & 1987 & 1988 \\
\hline $\begin{array}{l}10 \\
10\end{array}$ & $\begin{array}{l}\mathrm{BI}(12.85 \%) \\
\operatorname{PR}(39.31 \%)\end{array}$ & $\begin{array}{l}\mathrm{BI}(11.33 \%) \\
\operatorname{PR}(37.45 \%)\end{array}$ & $\begin{array}{l}\mathrm{BI}(12.44 \%) \\
\operatorname{PR}(42.09 \%)\end{array}$ & $\begin{array}{l}\mathrm{BI}(14.79 \%) \\
\operatorname{PR}(21.65 \%)\end{array}$ & $\begin{array}{l}\text { BI. }(14.30 \%) \\
\text { PR }(32.41 \%)\end{array}$ \\
\hline $\begin{array}{l}11 \\
11\end{array}$ & $\begin{array}{l}\mathrm{BI} \quad(8.65 \%) \\
\operatorname{PR}(32.88 \%)\end{array}$ & $\begin{array}{l}\mathrm{BI} \quad(8.97 \%) \\
\operatorname{PR}(29.66 \%)\end{array}$ & $\begin{array}{l}\mathrm{BI} \quad(7.44 \%) \\
\operatorname{PR}(30.37 \%)\end{array}$ & $\begin{array}{l}\mathrm{BI} \quad(6.76 \%) \\
\mathrm{PR}(38.23 \%)\end{array}$ & $\begin{array}{l}\mathrm{BI} \quad(6.87 \%) \\
\operatorname{PR}(35.36 \%)\end{array}$ \\
\hline $\begin{array}{l}12 \\
12\end{array}$ & $\begin{array}{l}\mathrm{BI}(25.24 \%) \\
\operatorname{PR}(25.88 \%)\end{array}$ & $\begin{array}{l}\mathrm{BI}(18.52 \%) \\
\operatorname{PR}(28.90 \%)\end{array}$ & $\begin{array}{l}\mathrm{BI}(16.29 \%) \\
\operatorname{PR}(38.29 \%)\end{array}$ & $\begin{array}{l}\mathrm{BI}(19.20 \%) \\
\operatorname{PR}(29.27 \%)\end{array}$ & $\begin{array}{l}\mathrm{Bl}(21.02 \%) \\
A D(29.21 \%)\end{array}$ \\
\hline $\begin{array}{l}13 \\
13\end{array}$ & $\begin{array}{l}\mathrm{BI}(14.96 \%) \\
\mathrm{AD}(44.25 \%)\end{array}$ & $\begin{array}{l}\mathrm{BI}(15.38 \%) \\
\mathrm{AD}(45.96 \%)\end{array}$ & $\begin{array}{l}\mathrm{BI}(13.19 \%) \\
\operatorname{PR}(42.51 \%)\end{array}$ & $\begin{array}{l}\mathrm{BI}(12.82 \%) \\
\mathrm{AD}(42.21 \%)\end{array}$ & $\begin{array}{l}\mathrm{BI}(12.81 \%) \\
\mathrm{AD}(43.33 \%)\end{array}$ \\
\hline $\begin{array}{l}15 \\
15\end{array}$ & $\begin{array}{l}\text { BI }(18.88 \%) \\
\text { PT }(50.26 \%)\end{array}$ & $\begin{array}{l}\mathrm{BI}(16.12 \%) \\
\mathrm{PT}(36.32 \%)\end{array}$ & $\begin{array}{l}\mathrm{BI}(18.16 \%) \\
\text { PT (35.96\%) }\end{array}$ & $\begin{array}{l}\text { BI }(20.01 \%) \\
\text { PT }(35.04 \%)\end{array}$ & $\begin{array}{l}\mathrm{BI}(18.68 \%) \\
\text { PT }(35.93 \%)\end{array}$ \\
\hline $\begin{array}{l}16 \\
16\end{array}$ & $\begin{array}{l}\mathrm{BI}(39.86 \%) \\
\mathrm{BI}(39.86 \%)\end{array}$ & $\begin{array}{l}\mathrm{BI}(35.31 \%) \\
\mathrm{BI}(35.31 \%)\end{array}$ & $\begin{array}{l}\mathrm{BI}(30.06 \%) \\
\operatorname{PR}(30.74 \%)\end{array}$ & $\begin{array}{l}\mathrm{BI}(26.05 \%) \\
\operatorname{PR}(30.40 \%)\end{array}$ & $\begin{array}{l}\mathrm{BI}(19.26 \%) \\
\operatorname{PR}(32.12 \%)\end{array}$ \\
\hline $\begin{array}{l}17 \\
17\end{array}$ & $\begin{array}{l}\text { BI }(15.84 \%) \\
\text { PT }(32.17 \%)\end{array}$ & $\begin{array}{l}\mathrm{BI}(12.67 \%) \\
\mathrm{AD}(25.71 \%)\end{array}$ & $\begin{array}{l}\mathrm{BI}(13.30 \%) \\
\mathrm{AD}(29.44 \%)\end{array}$ & $\begin{array}{l}\mathrm{BI}(13.49 \%) \\
\mathrm{AD}(25.97 \%)\end{array}$ & $\begin{array}{l}\mathrm{BI}(13.55 \%) \\
A D(23.93 \%)\end{array}$ \\
\hline $\begin{array}{l}18 \\
18\end{array}$ & $\begin{array}{l}\text { BI }(9.43 \%) \\
\text { PT }(35.98 \%)\end{array}$ & $\begin{array}{l}\mathrm{BI}(10.08 \%) \\
\mathrm{PT}(30.13 \%)\end{array}$ & $\begin{array}{l}\mathrm{BI}(12.92 \%) \\
\mathrm{PT}(37.45 \%)\end{array}$ & $\begin{array}{l}\text { BI }(13.43 \%) \\
\text { PT }(35.09 \%)\end{array}$ & $\begin{array}{l}\mathrm{BI}(12.88 \%) \\
\operatorname{PT}(35.36 \%)\end{array}$ \\
\hline $\begin{array}{l}19 \\
19\end{array}$ & $\begin{array}{l}\text { BI }(27.14 \%) \\
\text { PT }(29.71 \%)\end{array}$ & $\begin{array}{l}\mathrm{BI}(27.07 \%) \\
\mathrm{BI}(27.07 \%)\end{array}$ & $\begin{array}{l}\mathrm{BI}(28.52 \%) \\
\mathrm{BI}(28.52 \%)\end{array}$ & $\begin{array}{l}\mathrm{BI}(28.87 \%) \\
\mathrm{BI}(28.87 \%)\end{array}$ & $\begin{array}{l}\mathrm{BI}(30.32 \%) \\
\mathrm{BI}(30.32 \%)\end{array}$ \\
\hline $\begin{array}{l}20 \\
20\end{array}$ & $\begin{array}{l}\mathrm{BI}(12.03 \%) \\
\mathrm{AD}(34.73 \%)\end{array}$ & $\begin{array}{l}\mathrm{BI}(11.92 \%) \\
\mathrm{AD}(29.96 \%)\end{array}$ & $\begin{array}{l}\mathrm{BI}(12.38 \%) \\
\mathrm{AD}(33.38 \%)\end{array}$ & $\begin{array}{l}\mathrm{BI}(11.36 \%) \\
\operatorname{AD}(26.32 \%)\end{array}$ & $\begin{array}{l}\mathrm{BI}(15.21 \%) \\
\mathrm{AD}(22.97 \%)\end{array}$ \\
\hline $\begin{array}{l}24 \\
24\end{array}$ & $\begin{array}{l}\mathrm{BI} \quad(9.28 \%) \\
\operatorname{PR}(34.88 \%)\end{array}$ & $\begin{array}{l}\text { BI }(10.65 \%) \\
\operatorname{PR}(33.38 \%)\end{array}$ & $\begin{array}{l}\text { BI }(11.43 \%) \\
\text { PT }(33.01 \%)\end{array}$ & $\begin{array}{l}\mathrm{BI}(11.95 \%) \\
\operatorname{PR}(32.26 \%)\end{array}$ & $\begin{array}{l}\mathrm{BI}(10.97 \%) \\
\operatorname{PR}(35.47 \%)\end{array}$ \\
\hline $\begin{array}{l}25 \\
25\end{array}$ & $\begin{array}{l}\mathrm{BI}(7.38 \%) \\
\mathrm{CC}(56.16 \%)\end{array}$ & $\begin{array}{l}\mathrm{BI}(36.55 \%) \\
\mathrm{CC}(46.66 \%)\end{array}$ & $\begin{array}{l}\mathrm{BI}(23.61 \%) \\
\operatorname{PR}(33.07 \%)\end{array}$ & $\begin{array}{l}\mathrm{BI}(38.23 \%) \\
\operatorname{PR}(55.84 \%)\end{array}$ & $\begin{array}{l}\mathrm{BI}(18.21 \%) \\
\text { PT }(39.61 \%)\end{array}$ \\
\hline $\begin{array}{l}27 \\
27\end{array}$ & $\begin{array}{l}\text { BI }(16.62 \%) \\
\text { PT }(33.62 \%)\end{array}$ & $\begin{array}{l}\text { BI }(14.42 \%) \\
\text { PT }(33.46 \%)\end{array}$ & $\begin{array}{l}\text { BI }(13.34 \%) \\
\text { PT }(28.64 \%)\end{array}$ & $\begin{array}{l}\text { BI }(13.42 \%) \\
\text { PT }(28.72 \%)\end{array}$ & $\begin{array}{l}\text { BI }(14.96 \%) \\
\text { PT }(28.36 \%)\end{array}$ \\
\hline $\begin{array}{l}28 \\
28\end{array}$ & $\begin{array}{l}\mathrm{BI}(19.47 \%) \\
\operatorname{AD}(28.75 \%)\end{array}$ & $\begin{array}{l}B I(11.03 \%) \\
A D(26.51 \%)\end{array}$ & $\begin{array}{l}\mathrm{BI} \quad(9.07 \%) \\
\mathrm{PS}(26.98 \%)\end{array}$ & $\begin{array}{l}\mathrm{BI} \quad(9.76 \%) \\
\operatorname{PR}(25.80 \%)\end{array}$ & $\begin{array}{l}\mathrm{BI} \quad(9.90 \%) \\
A D(25.39 \%)\end{array}$ \\
\hline $\begin{array}{l}29 \\
29\end{array}$ & $\begin{array}{l}\text { BI }(18.06 \%) \\
\text { PT }(26.21 \%)\end{array}$ & $\begin{array}{l}\text { BI }(15.26 \%) \\
\text { PT }(23.84 \%)\end{array}$ & $\begin{array}{l}\text { BI }(11.91 \%) \\
\text { PT }(23.89 \%)\end{array}$ & $\begin{array}{l}\mathrm{BI}(11.65 \%) \\
\mathrm{AD}(26.05 \%)\end{array}$ & $\begin{array}{l}B I(13.33 \%) \\
A D(24.52 \%)\end{array}$ \\
\hline $\begin{array}{l}31 \\
31\end{array}$ & $\begin{array}{l}\mathrm{BI} \quad(9.39 \%) \\
\text { PT }(38.76 \%)\end{array}$ & $\begin{array}{l}\text { BI. }(9.57 \%) \\
\text { PT }(40.02 \%)\end{array}$ & $\begin{array}{l}\text { BI }(9.15 \%) \\
\text { PT }(39.35 \%)\end{array}$ & $\begin{array}{l}\text { BI }(6.13 \%) \\
\text { PT }(39.14 \%)\end{array}$ & $\begin{array}{l}\text { BI }(9.21 \%) \\
\text { PT }(36.59 \%)\end{array}$ \\
\hline $\begin{array}{l}34 \\
34\end{array}$ & $\begin{array}{l}\text { BI }(11.43 \%) \\
\text { PT }(36.08 \%)\end{array}$ & $\begin{array}{l}\mathrm{BI}(12.38 \%) \\
\text { PT }(31.84 \%)\end{array}$ & $\begin{array}{l}\text { BI }(12.81 \%) \\
\text { PT }(33.51 \%)\end{array}$ & $\begin{array}{l}\text { BI }(11.59 \%) \\
\text { PT }(27.87 \%)\end{array}$ & $\begin{array}{l}\text { BI }(11.41 \%) \\
\text { PT }(27.82 \%)\end{array}$ \\
\hline $\begin{array}{l}35 \\
35\end{array}$ & $\begin{array}{l}\mathrm{BI} \quad(7.37 \%) \\
\mathrm{PT}(43.78 \%)\end{array}$ & $\begin{array}{l}\mathrm{BI} \quad(7.41 \%) \\
\text { PT }(43.27 \%)\end{array}$ & $\begin{array}{l}\text { BI }(7.61 \%) \\
\text { PT }(42.13 \%)\end{array}$ & $\begin{array}{l}\text { BI }(9.34 \%) \\
\text { PT }(36.65 \%)\end{array}$ & $\begin{array}{l}\mathrm{BI} \quad(9.14 \%) \\
\operatorname{PR}(37.49 \%)\end{array}$ \\
\hline $\begin{array}{l}38 \\
38\end{array}$ & $\begin{array}{l}\mathrm{BI}(4.29 \%) \\
\mathrm{AD}(44.19 \%)\end{array}$ & $\begin{array}{l}\mathrm{BI}(11.13 \%) \\
\mathrm{AD}(33.96 \%)\end{array}$ & $\begin{array}{l}\mathrm{BI}(21.25 \%) \\
\operatorname{PR}(29.89 \%)\end{array}$ & $\begin{array}{l}\mathrm{BI}(16.55 \%) \\
\mathrm{AD}(32.56 \%)\end{array}$ & $\begin{array}{l}\mathrm{BI}(14.09 \%) \\
\mathrm{AD}(36.05 \%)\end{array}$ \\
\hline $\begin{array}{l}40 \\
40\end{array}$ & $\begin{array}{l}\text { BI }(11.90 \%) \\
\operatorname{PR}(26.04 \%)\end{array}$ & $\begin{array}{l}\mathrm{BI}(12.03 \%) \\
\operatorname{PR}(25.18 \%)\end{array}$ & $\begin{array}{l}\text { BI }(13.99 \%) \\
\text { PT }(22.98 \%)\end{array}$ & $\begin{array}{l}\mathrm{BI}(14.82 \%) \\
\operatorname{PR}(24.00 \%)\end{array}$ & $\begin{array}{l}\mathrm{BI}(14.05 \%) \\
\operatorname{PR}(31.40 \%)\end{array}$ \\
\hline $\begin{array}{l}41 \\
41\end{array}$ & $\begin{array}{l}\mathrm{BI}(15.67 \%) \\
\mathrm{PT}(34.17 \%)\end{array}$ & $\begin{array}{l}\text { BI }(17.99 \%) \\
\text { PT }(33.34 \%)\end{array}$ & $\begin{array}{l}\text { BI }(17.01 \%) \\
\text { PT }(28.82 \%)\end{array}$ & $\begin{array}{l}\text { BI (20.77\%) } \\
\text { PT (32.91\%) }\end{array}$ & $\begin{array}{l}\text { BI }(25.40 \%) \\
\text { PT }(32.47 \%)\end{array}$ \\
\hline $\begin{array}{r}42 \\
42\end{array}$ & $\begin{array}{l}\text { BI }(16.35 \%) \\
\text { PT }(40.34 \%)\end{array}$ & $\begin{array}{l}\text { BI }(12.21 \%) \\
\text { PT }(35.67 \%)\end{array}$ & $\begin{array}{l}\mathrm{BI}(12.71 \%) \\
\text { PT }(34.47 \%)\end{array}$ & $\begin{array}{l}\mathrm{BI}(13.45 \%) \\
\text { PT }(34.34 \%)\end{array}$ & $\begin{array}{l}\text { BI }(13.51 \%) \\
\text { PT }(30.70 \%)\end{array}$ \\
\hline
\end{tabular}


le taux ne put être calculé. Pour leur part, les Parcs ont connu 66 fois une croissance $(+)$ et 22 fois une décroissance (-). Par contre il est à noter que les Bibliothèques ont connu 79 fois une croissance $(+)$ de leur budget de dépenses et seulement 9 fois une décroissance (-).

Mais afin de mieux visualiser ces changements, trois tableaux 10, 11 et 12, (voir NDLR) représentent graphiquement les données (croissances et décroissances) du tableau 9.

À notre avis, ces tableaux montrent de façon assez claire « les gestes posés et les décisions prises " en ce qui a trait à ces trois postes budgétaires du secteur Loisirs et Culture, soit les Patinoires, les Parcs et les Bibliothèques. À vrai dire, ces représentations graphiques font ressortir certaines différences moins facilement décelables au tableau précédent. Par exemple, les parcs et terrains de jeux semblent être les plus exposés aux changements soudains de cap, tandis que pour les bibliothèques, ces changements, même de moindre ampleur, se caractérisent davantage par une croissance peu élevée mais continue. II faut cependant garder à l'esprit les mises en garde de la Commission d'étude sur les municipalités. Ces changements, calculés à partir des dépenses consenties, reflètent "bien plus les coûts de ces services que la quantité produite ou leur qualité ".

Avant de conclure sur ces quelques données, certaines mises au point s'imposent. Premièrement, il est bon de se rappeler que la période étudiée fut une période relativement stable et prospère du point de vue économique. Deuxièmement, bien que la présente analyse ne repose que sur les données financières de 22 municipalités, au point de départ celles-ci ont été choisies au hasard et bien que leur nombre ait été réduit de 35 à 22, elles continuent de représenter des municipalités de tailles diverses réparties sur tout le territoire québécois. Troisièmement, en 1986, une décision ministérielle émanant du ministère des Affaires culturelles venait quelque peu perturber le développement des bibliothèques publiques ${ }^{18}$.

Si l'on se reporte aux résultats de l'évaluation des perceptions présentés au début de cet article, un tel incident peut expliquer, du moins en partie, l'importance minimale que l'adminis- tration municipale semble attribuer à "Consulter les représentants du ministère des Affaires culturelles responsables du développement des bibliothèques publiques" (tableau 3, \#46). Aussi, le dernier volet de notre analyse du support financier consenti à la bibliothèque consiste à examiner les changements survenus dans les subventions du ministère des Affaires culturelles. À notre avis, les tableaux 13 et 14 (voir NDLR) illustrent bien la décision ministérielle de 1986.

\section{Perception et support financier}

Dans un contexte d'intégration municipale, les porte-parole officiels de la bibliothèque auprès du conseil municipal sont peu nombreux. Selon la hiérarchie administrative, au plus trois ou quatre personnes sont mandatées pour expliquer aux décideurs politiques la raison d'être, le fonctionnement et les besoins d'une telle institution. Par contre ce sont ces décideurs politiques qui allouent le support financier nécessaire au développement et au bon fonctionnement de la bibliothèque ${ }^{19}$.

D'autre part, les élus municipaux ont la responsabilité d'administrer plusieurs autres "dossiers municipaux". Des parcs et terrains de jeux au Service de la police, le champ des responsabilités est vaste.

La principale question sous-tendant cette recherche est la suivante: "Dans quelle mesure l'administration municipale apporte-t-elle son support au 'dossier bibliothèque'?" Afin de répondre à cette question, deux approches analytiques furent proposées: I'une en termes d'évaluation des perceptions et l'autre en termes de support financier.

La première analyse démontra qu'en ce qui a trait à l'importance des divers éléments qui définissent une bibliothèque publique, peu d'éléments forment un consensus entre les divers groupes de l'administration municipale concernés par ce dossier. La deuxième analyse démontra que le support financier, bien que constant et en croissance, semble occuper une place relativement minime parmi les dépenses municipales auxquelles participe la bibliothèque.

La dernière question que l'on doit se poser est: "Existe-t-il un lien entre 'perception' et 'support financier' ?" C'est-à-dire, "Des différences de perceptions au sein de l'administration municipale expliquent-elles, du moins en partie, le niveau de support financier accordé au dossier bibliothèque? "

Dans un milieu politique comme le milieu municipal, plusieurs facteurs influencent les décisions. Certains de ces facteurs sont étroitement liés à I'environnement, donc extérieurs à l'administration municipale, tandis que d'autres sont internes et liés au fonctionnement de l'appareil administratif municipal lui-même. Par contre plusieurs de ces facteurs s'entremêlent et une évaluation de l'influence de chacun sur le processus décisionnel des élus municipaux peut s'avérer un exercice fort complexe.

À titre d'exemple, nous ne mentionnons que quelques-uns de ces facteurs. Ainsi, parmi les facteurs externes on retrouve en tout premier lieu le gouvernement provincial. Comme on a pu le constater depuis quelques mois, les décisions et les agissements du gouvernement provincial influencent dans une très large mesure certaines décisions du milieu municipal 20 . Un autre facteur externe concerne les ressources financières dont dispose la municipalité. Comme le montre le débat des Assises Québecmunicipalités, le contrôle de ce facteur réside le plus souvent à l'extérieur de l'administration municipale. II va sans dire que la municipalité dispose de plus ou moins de ressources financières et jouit d'une plus ou moins grande capacité de les augmenter. Elle doit donc tenir compte de ce facteur dans ses décisions. Un troisième facteur, qui est très souvent extérieur à l'administration municipale, est la nature des services ou des secteurs de services que la municipalité est tenue d'offrir à ses citoyens. Certains services sont obligatoires et, de plus,

NDLR: Les tableaux $10,11,12$ et 14 n'ont pas été reproduits dans le cadre de cet article mais peuvent être consultés au secrétariat de I'ASTED. Ils ont été joints au rapport de recherche de l'auteure.

18. Lise Caron, "Le rapport financier $1990 \ldots$..., 9.

19. Diane Mittermeyer, "Le développement des bibliothèques publiques du Québec: un pas en avant, deux pas en arrière", Argus, vol. 17, no 2 (1988), 37-46.

20. Diane Mittermeyer, "La bibliothèque publique et le milieu....», 147 . 
hautement réglementés. Si la municipalité n'a que peu de latitude dans la décision d'offrir certains services et d'en augmenter le niveau, ce peu de latitude influence nécessairement la prise de décisions dans d'autres domaines comme, par exemple, la quantité, la qualité, et les coûts d'autres services municipaux non soumis à de telles exigences.

Le dernier facteur externe à l'administration municipale que nous aimerions souligner est la demande des citoyens. Surtout en ce qui concerne la manière dont cette demande est exprimée et la nature des services auxquels ces demandes se réfèrent. Lorsque I'Association des citoyens réclame un meilleur éclairage des rues en en faisant une question de sécurité publique, une telle demande est susceptible d'influencer l'administration municipale de façon plus efficace que la demande du club de tennis local qui désire un agrandissement des terrains mis à sa disposition.

Ce facteur de la demande des citoyens, bien que souvent moins tangible que les facteurs précédents, est souvent celui qui fait la différence en termes de quantité, de qualité et de coûts consentis. II ne faut pas oublier que les élus municipaux, aux prises avec toutes les exigences qu'on leur impose, ne possèdent souvent qu'un mince champ de manoeuvre pour répondre aux nombreuses demandes de leurs citoyens. Pourtant, en bout de ligne, c'est ce facteur de satisfaction qui risque d'influencer le prochain scrutin.

Parmi les facteurs internes, on retrouve en premier lieu la hiérarchie administrative. Tout d'abord il faut noter le rôle central du directeur général. Par définition, c'est lui qui supervise l'ensemble des employés et des services municipaux. II est le lien direct, reconnu et mandaté entre élus et employés, donc entre ceux qui décident des services à offrir et qui allouent les fonds nécessaires et ceux qui exécutent les décisions selon les moyens et le support qu'on leur donne. Une des responsabilités majeures du directeur général est donc $d$ 'analyser et d'évaluer les demandes et les recommandations qui sont adressées au conseil afin de lui faciliter la prise de décisions. II faut aussi noter que dans une large proportion, lorsque ces demandes, suggestions et recom- mandations sont soumises au directeur général pour être ensuite acheminées au conseil, elles ont déjà été analysées et évaluées par un directeur de service. La hiérarchie administrative joue donc un rôle important dans la prise de décisions des élus.

De cette hiérarchie découle un second facteur susceptible d'influencer la prise de décision. II s'agit d'un élément de compétition au sein même de l'administration, entre les divers services qui, somme toute, rivalisent pour l'obtention de ressources financières qui restent de plus en plus limitées. Si une saine concurrence est normale et même stimulante, il n'en: demeure pas moins que ce facteur peut jouer un rôle déterminant dans la prise de certaines décisions. Cette compétition se situe davantage sur un plan administratif que sur un plan personnel entre directeurs de services, bien que ce dernier aspect ne soit pas négligeable. À quelques reprises nous avons eu l'occasion de constater que "la petite fille de la bibliothèque ", en l'occurence la directrice de ce service municipal, ne faisait que difficilement le poids (au propre et au figuré) face au gérant de l'aréna ou au directeur des travaux publics.

En dernier lieu, nous aimerions souligner un facteur à la fois interne et externe, celui de la visibilité du service. Bien que tous les services offerts par une municipalité soient, par définition; jugés nécessaires à la "qualité de vie" d'une communauté donnée, il va sans dire que tous ces services n'ont pas la même visibilité, et ce, aussi bien pour l'administration municipale (interne) que pour les citoyens (externe). Par exemple, dans le secteur Loisirs et Culture, une patinoire tout en creux et en bosses, ou un terrain de balles qui s'assèche mal et qúi est constamment boueux risque, si la situation persiste, de faire plus de bruit à la réunion du conseil municipal que l'entassement de documents décrépits sur les rayons de la bibliothèque. Ce genre de visibilité sert également bien la concurrence entre les services.

Ce bref aperçu des nombreux facteurs pouvant influencer les décisions des élus municipaux illustre, dans une certaine mesure, la complexité du processus décisionnel municipal.

\section{Et la bibliothèque !}

Si dans notre esprit il ne fait aucun doute que la bibliothèque soit un service municipal à part entière, nous nous devons d'ajouter: "OUI, MAIS...".

Dans l'éventualité d'une généralisation des résultats de la présente étude, nous avons tenté d'identifier parmi les facteurs mentionnés précédemment ceux qui pourraient influencer de façon positive les décisions prises par le conseil municipal.

Certains agissements récents du gouvernement provincial seraient de nature à semer le doute plutôt que la confiance et la motivation. À ce sujet, les propos de Maud Lefebvre-Roux, sont révélateurs:

Une fois de plus, nos subventions nous arrivent sur le tard et amputées de quelques milliers de dollars. En effet, malgré le ton bienveillant de Madame la ministre, il s'agit bel et bien d'une "coupure" qui survient alors que les trois quarts de l'année financière sont entamés. Cela ne représente que $1,9 \%$, nous répond la direction des bibliothèques. Alors que pour certains l'impact est mineur, pour d'autres, il s'agit d'une véritable catastrophe et la guerre des chiffres est engagée...

Pourtant, au-delà du manque à gagner que cette décision arbitraire crée pour nos municipalités, on craint d'entacher la crédibilité des directeurs de bibliothèques en tant que gestionnaires. Mais n'est-ce pas plutôt la crédibilité du ministère des Affaires culturelles qui doit être remise en question plutôt que la capacité du directeur à bien gérer le budget de sa bibliothèque.

Le ministère des Affaires culturelles par le biais de la Direction des bibliothèques publiques propose un programme $d^{\prime}$ aide financière au développement des bibliothèques publiques autonomes à ce point intéressant qu'un nombre important de municipalités s'y inscrivent. Madame la ministre constate "qu'encore cette année, ce nouveau programme d'aide fait l'objet d'un intérêt marqué de la part des municipalités..." ". Le ministère répond à cét engouement en procédant à des coupures de subventions, le tout 


\begin{tabular}{|c|c|c|c|c|c|}
\hline \multicolumn{6}{|c|}{$\begin{array}{l}\text { Tableau } 9 \\
\text { Changements }(+/-) \text { du budget de dépenses pour les postes Patinoires, Parcs, Bibliothèques } \\
\text { (Année de base : 1984) }\end{array}$} \\
\hline Municipalités & Poste & 1985 & 1986 & 1987 & 1988 \\
\hline 10 & $\begin{array}{l}\text { Patinoires } \\
\text { Parcs } \\
\text { Bibliothèques }\end{array}$ & $\begin{array}{l}+30.3 \% \\
+57.0 \% \\
+\quad 45.3 \%\end{array}$ & $\begin{array}{l}\quad \overline{-}^{*} \\
+\quad 11.5 \% \\
+\quad 8.9 \%\end{array}$ & $\begin{array}{r}- \\
-\quad 52.5 \% \\
+\quad 9.8 \%\end{array}$ & $\begin{array}{r}- \\
+58.0 \% \\
+\quad 2.0 \%\end{array}$ \\
\hline 11 & $\begin{array}{l}\text { Patinoires } \\
\text { Parcs } \\
\text { Bibliothèques }\end{array}$ & $\begin{array}{l}+15.7 \% \\
+31.6 \% \\
+51.2 \%\end{array}$ & $\begin{array}{l}+10.4 \% \\
+\quad 8.2 \% \\
-12.3 \%\end{array}$ & $\begin{array}{l}-18.6 \% \\
+\quad 47.1 \% \\
+\quad 6.3 \%\end{array}$ & $\begin{array}{l}+\quad 10.2 \% \\
-\quad 5.9 \% \\
+\quad 3.3 \%\end{array}$ \\
\hline 12 & $\begin{array}{l}\text { Patinoires } \\
\text { Parcs } \\
\text { Bibliothèques }\end{array}$ & $\begin{array}{l}+\quad 46.1 \% \\
+\quad 54.9 \% \\
+\quad 1.8 \%\end{array}$ & $\begin{array}{l}+14.9 \% \\
+\quad 75.1 \% \\
+\quad 16.2 \%\end{array}$ & $\begin{array}{l}+12.2 \% \\
-\quad 28.0 \% \\
+\quad 11.0 \%\end{array}$ & $\begin{array}{r}+\quad 10.4 \% \\
-\quad 1.9 \% \\
+\quad 17.4 \%\end{array}$ \\
\hline 13 & $\begin{array}{l}\text { Patinoires } \\
\text { Parcs } \\
\text { Bibliothèques }\end{array}$ & $\begin{array}{l}-\quad 9.3 \% \\
+\quad 37.3 \% \\
+\quad 2.7 \%\end{array}$ & $\begin{array}{l}-\quad 29.1 \% \\
+\quad 25.3 \% \\
+\quad 8.1 \%\end{array}$ & $\begin{array}{l}+206.1 \% \\
+\quad 20.2 \% \\
+\quad 5.5 \%\end{array}$ & $\begin{array}{l}-\quad 3.2 \% \\
+\quad 7.4 \% \\
+\quad 6.7 \%\end{array}$ \\
\hline 15 & $\begin{array}{l}\text { Patinoires } \\
\text { Parcs } \\
\text { Bibliothèques }\end{array}$ & $\begin{array}{l}+11.6 \% \\
+102.8 \% \\
+31.9 \%\end{array}$ & $\begin{array}{l}+\quad 5.3 \% \\
-\quad 4.6 \% \\
+\quad 19.7 \%\end{array}$ & $\begin{array}{r}+\quad 1.4 \% \\
-\quad 1.7 \% \\
+\quad 14.7 \%\end{array}$ & $\begin{array}{l}+\quad 9.0 \% \\
+\quad 2.7 \% \\
-\quad 0.7 \%\end{array}$ \\
\hline 16 & $\begin{array}{l}\text { Patinoires } \\
\text { Parcs } \\
\text { Bibliothèques }\end{array}$ & $\begin{array}{l}-16.5 \% \\
+\quad 92.7 \% \\
+\quad 74.3 \%\end{array}$ & $\begin{array}{l}+\quad 96.1 \% \\
+\quad 37.8 \% \\
+\quad 6.5 \%\end{array}$ & $\begin{array}{r}+\quad 6.3 \% \\
+\quad 24.8 \% \\
+\quad 9.4 \%\end{array}$ & $\begin{array}{l}+63.6 \% \\
+58.0 \% \\
+\quad 10.5 \%\end{array}$ \\
\hline 17 & $\begin{array}{l}\text { Patinoires } \\
\text { Parcs } \\
\text { Bibliothèques }\end{array}$ & $\begin{array}{l}+14.5 \% \\
+\quad 46.4 \% \\
+\quad 18.6 \%\end{array}$ & $\begin{array}{l}-\quad 3.0 \% \\
+\quad 82.5 \% \\
+\quad 14.7 \%\end{array}$ & $\begin{array}{l}+\quad 5.2 \% \\
+\quad 23.8 \% \\
+\quad 3.4 \%\end{array}$ & $\begin{array}{r}-10.8 \% \\
+\quad 18.6 \% \\
+\quad 0.6 \%\end{array}$ \\
\hline 18 & $\begin{array}{l}\text { Patinoires } \\
\text { Parcs } \\
\text { Bibliothèques }\end{array}$ & $\begin{array}{l}+\quad 13.4 \% \\
+\quad 8.2 \% \\
+\quad 44.8 \%\end{array}$ & $\begin{array}{l}+\quad 1.1 \% \\
+\quad 5.8 \% \\
+\quad 4.2 \%\end{array}$ & $\begin{array}{l}-\quad 4.1 \% \\
+\quad 9.5 \% \\
+\quad 6.4 \%\end{array}$ & $\begin{array}{l}+\quad 13.8 \% \\
+\quad 28.0 \% \\
+\quad 3.3 \%\end{array}$ \\
\hline 19 & $\begin{array}{l}\text { Patinoires } \\
\text { Parcs } \\
\text { Bibliothèques }\end{array}$ & $\begin{array}{l}+\quad 9.1 \% \\
+\quad 13.7 \% \\
+\quad 24.4 \%\end{array}$ & $\begin{array}{l}+\quad 9.7 \% \\
+\quad 26.0 \% \\
+\quad 19.6 \%\end{array}$ & $\begin{array}{l}+\quad 26.4 \% \\
+\quad 11.8 \% \\
+\quad 28.7 \%\end{array}$ & $\begin{array}{l}+\quad 0.4 \% \\
-\quad 7.2 \% \\
+\quad 20.5 \%\end{array}$ \\
\hline 20 & $\begin{array}{l}\text { Patinoires } \\
\text { Parcs } \\
\text { Bibliothèques }\end{array}$ & $\begin{array}{l}+\quad 8.5 \% \\
+\quad 39.8 \% \\
+\quad 24.2 \%\end{array}$ & $\begin{array}{l}+\quad 11.1 \% \\
-\quad 23.8 \% \\
+\quad 1.0 \%\end{array}$ & $\begin{array}{r}+\quad 54.1 \% \\
+\quad 14.8 \% \\
+\quad 2.6 \%\end{array}$ & $\begin{array}{r}-31.1 \% \\
-\quad 32.4 \% \\
-\quad 1.3 \%\end{array}$ \\
\hline 24 & $\begin{array}{l}\text { Patinoires } \\
\text { Parcs } \\
\text { Bibliothèques }\end{array}$ & $\begin{array}{l}+\quad 16.5 \% \\
+\quad 9.7 \% \\
+\quad 31.5 \%\end{array}$ & $\begin{array}{l}-\quad 0.2 \% \\
-\quad 6.0 \% \\
+\quad 6.5 \%\end{array}$ & $\begin{array}{r}+\quad 1.1 \% \\
+\quad 8.1 \% \\
+\quad 10.7 \%\end{array}$ & $\begin{array}{r}-\quad 0.5 \% \\
+\quad 33.7 \% \\
+\quad 11.7 \%\end{array}$ \\
\hline 25 & $\begin{array}{l}\text { Patinoires } \\
\text { Parcs } \\
\text { Bibliothèques }\end{array}$ & $\begin{array}{r}-{ }^{* *} \\
-\quad 89.2 \% \\
+\quad 16.5 \%\end{array}$ & $\begin{array}{r}-\overline{-} \\
+294.2 \% \\
+\quad 29.2 \%\end{array}$ & $\begin{aligned} & - \\
+ & 35.3 \% \\
+ & 29.8 \%\end{aligned}$ & $\begin{array}{r}-\overline{-} \\
+25.3 \% \\
+62.5 \%\end{array}$ \\
\hline 27 & $\begin{array}{l}\text { Patinoires } \\
\text { Parcs } \\
\text { Bibliothèques }\end{array}$ & $\begin{array}{l}+\quad 27.7 \% \\
-\quad 4.2 \% \\
+\quad 11.3 \%\end{array}$ & $\begin{array}{l}+\quad 0.7 \% \\
+\quad 61.6 \% \\
+\quad 8.8 \%\end{array}$ & $\begin{array}{l}+\quad 8.0 \% \\
+\quad 7.5 \% \\
+\quad 8.4 \%\end{array}$ & $\begin{array}{l}+\quad 2.0 \% \\
+\quad 12.5 \% \\
+\quad 15.2 \%\end{array}$ \\
\hline 28 & $\begin{array}{l}\text { Patinoires } \\
\text { Parcs } \\
\text { Bibliothèques }\end{array}$ & $\begin{array}{l}+185.1 \% \\
+\quad 42.6 \% \\
+\quad 21.1 \%\end{array}$ & $\begin{array}{l}+\quad 0.3 \% \\
+\quad 36.3 \% \\
-\quad 0.8 \%\end{array}$ & $\begin{array}{l}+\quad 15.9 \% \\
+110.6 \% \\
+\quad 14.0 \%\end{array}$ & $\begin{array}{l}-19.6 \% \\
-\quad 9.8 \% \\
+\quad 5.2 \%\end{array}$ \\
\hline 29 & $\begin{array}{l}\text { Patinoires } \\
\text { Parcs } \\
\text { Bibliothèques }\end{array}$ & $\begin{array}{l}+\quad 48.1 \% \\
+645.3 \% \\
+\quad 37.9 \%\end{array}$ & $\begin{array}{r}+\quad 6.0 \% \\
+\quad 7.8 \% \\
-\quad 17.4 \%\end{array}$ & $\begin{array}{l}-\quad 1.0 \% \\
-\quad 4.6 \% \\
+\quad 0.7 \%\end{array}$ & $\begin{array}{l}+\quad 10.3 \% \\
+\quad 8.4 \% \\
+\quad 22.1 \%\end{array}$ \\
\hline
\end{tabular}




\begin{tabular}{|c|c|c|c|c|c|}
\hline \multicolumn{6}{|c|}{$\begin{array}{l}\text { Tableau } 9 \text { (suite) } \\
\text { Changements }(+/-) \text { du budget de dépenses pour les postes Patinoires, Parcs, Bibliothèques } \\
\text { (Année de base : 1984) }\end{array}$} \\
\hline Municipalités & Poste & 1985 & 1986 & 1987 & 1988 \\
\hline 31 & $\begin{array}{l}\text { Patinoires } \\
\text { Parcs } \\
\text { Bibliothèques }\end{array}$ & $\begin{array}{l}+\quad 21.8 \% \\
-\quad 8.1 \% \\
+\quad 14.1 \%\end{array}$ & $\begin{array}{l}+\quad 14.3 \% \\
+\quad 8.7 \% \\
+\quad 11.2 \%\end{array}$ & $\begin{array}{l}-\quad 4.2 \% \\
-\quad 1.8 \% \\
-35.5 \%\end{array}$ & $\begin{array}{l}+\quad 4.7 \% \\
+\quad 1.0 \% \\
+68.4 \%\end{array}$ \\
\hline 34 & $\begin{array}{l}\text { Patinoires } \\
\text { Parcs } \\
\text { Bibliothèques }\end{array}$ & $\begin{array}{l}+13.9 \% \\
+\quad 6.3 \% \\
+\quad 39.9 \%\end{array}$ & $\begin{array}{l}-\quad 0.9 \% \\
-\quad 22.6 \% \\
-\quad 2.6 \%\end{array}$ & $\begin{array}{l}+1.4 \% \\
+91.6 \% \\
+10.3 \%\end{array}$ & $\begin{array}{l}+20.7 \% \\
+21.3 \% \\
+19.1 \%\end{array}$ \\
\hline 35 & $\begin{array}{l}\text { Patinoires } \\
\text { Parcs } \\
\text { Bibliothèques }\end{array}$ & $\begin{array}{l}+\quad 3.6 \% \\
+\quad 0.5 \% \\
+\quad 5.4 \%\end{array}$ & $\begin{array}{l}+\quad 4.7 \% \\
+\quad 16.4 \% \\
+\quad 10.5 \%\end{array}$ & $\begin{array}{l}+\quad 5.6 \% \\
+32.3 \% \\
+49.0 \%\end{array}$ & $\begin{array}{l}+5.2 \% \\
+28.1 \% \\
+11.4 \%\end{array}$ \\
\hline 38 & $\begin{array}{l}\text { Patinoires } \\
\text { Parcs } \\
\text { Bibliothèques }\end{array}$ & $\begin{array}{l}+\quad 2.7 \% \\
+\quad 19.2 \% \\
+224.9 \%\end{array}$ & $\begin{array}{l}+18.4 \% \\
+31.9 \% \\
+141.1 \%\end{array}$ & $\begin{array}{l}-38.8 \% \\
-14.4 \% \\
-34.0 \%\end{array}$ & $\begin{array}{l}-\quad 4.3 \% \\
+\quad 3.3 \% \\
-\quad 9.2 \%\end{array}$ \\
\hline 40 & $\begin{array}{l}\text { Patinoires } \\
\text { Parcs } \\
\text { Bibliothèques }\end{array}$ & $\begin{array}{l}+11.7 \% \\
+\quad 10.8 \% \\
+\quad 15.8 \%\end{array}$ & $\begin{array}{l}+11.5 \% \\
+\quad 5.5 \% \\
+\quad 22.8 \%\end{array}$ & $\begin{array}{l}+\quad 9.1 \% \\
+22.9 \% \\
+22.3 \%\end{array}$ & $\begin{array}{l}+12.9 \% \\
+71.0 \% \\
+23.9 \%\end{array}$ \\
\hline 41 & $\begin{array}{l}\text { Patinoires } \\
\text { Parcs } \\
\text { Bibliothèques }\end{array}$ & $\begin{array}{l}+30.9 \% \\
+\quad 25.6 \% \\
+\quad 54.0 \%\end{array}$ & $\begin{array}{r}-\quad 7.4 \% \\
+\quad 17.1 \% \\
+\quad 1.3 \%\end{array}$ & $\begin{array}{l}+\quad 7.1 \% \\
+44.0 \% \\
+14.5 \%\end{array}$ & $\begin{array}{l}+\quad 3.0 \% \\
-16.6 \% \\
+27.7 \%\end{array}$ \\
\hline 42 & $\begin{array}{l}\text { Patinoires } \\
\text { Parcs } \\
\text { Bibliothèques }\end{array}$ & $\begin{array}{l}+35.9 \% \\
+109.3 \% \\
+\quad 14.7 \%\end{array}$ & $\begin{array}{l}-\quad 3.2 \% \\
-\quad 1.0 \% \\
+\quad 4.3 \%\end{array}$ & $\begin{array}{l}+\quad 9.0 \% \\
+\quad 5.6 \% \\
+15.7 \%\end{array}$ & $\begin{array}{l}+\quad 3.6 \% \\
+\quad 8.5 \% \\
+16.3 \%\end{array}$ \\
\hline * & 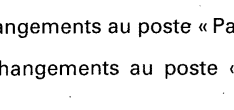 & 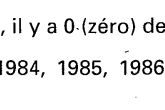 & 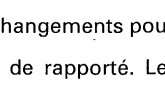 & . & $\begin{array}{l}\text { alculer. } \\
\text { ne peuvent }\end{array}$ \\
\hline
\end{tabular}

enrobé d'un discours politique quelque peu étriqué. II s'agit d'ailleurs de la deuxième édition de ce scénario puisqu'une situation similaire se produisait en $1986^{21}$.

En ce qui a trait aux ressources financières des municipalités ainsi qu'à la nature des différents secteurs de services municipaux, la nécessité de réviser les priorités pourrait également entraîner des prises de décisions pour le moins défavorables. La bibliothèque étant un service peu réglementé, donc n'imposant que des contraintes minimales, elle pourrait se retrouver au premier rang des services non prioritaires. Aux propos de Maud Lefebvre-Roux, il faut ajouter ceux de I'Union des municipalités du Québec:

Après des mois d'attente d'un projet gouvernemental qui devait être axé sur une véritable décentralisation des responsabilités, le résultat est amer : le ministre des Affaires municipales propose plutôt le transfert d'une facture d'au moins 500 millions de dollars aux municipalités ${ }^{22}$.

Les principaux services concernés, Transport en commun, Services policiers et Voirie locale, risquent fort de damer le pion à la bibliothèque. De plus, certains indices de l'évolution des finances municipales laissent croire que la bibliothèque pourrait connaître des changements susceptibles d'altérer son devenir. $\dot{A}$ titre d'exemple, nous aimerions signaler les deux indices suivants:

Le 24 août 1989 constitue une date importante dans l'histoire de la fiscalité municipale. C'est en effet depuis cette date que les municipalités peuvent exercer des pouvoirs très étendus en matière de tarification $^{23}$. et

L'UMO se félicite du fait que la ministre des Affaires culturelles, Mme Lucienne Robillard, ait décidé de ne pas déposer son projet de loi sur les bibliothèques publiques, qui empêchait la tarification de certains services offerts par les bibliothèques ${ }^{24}$.

21. Ralph Mercier, "Des chiffres et des lettres", Urba, vol. 12, no 1 (février 1991), 5. Voir auss dans ce numéro: "Assises Québecmunicipalités - La proposition gouvernementale: une facture d'au moins $500 \mathrm{M} \$$ ", 6-7, 22.

22. Maud Lefebvre-Roux, "Chers(ères) collègues", Défi, vol. 5, no 3 (décembre 1990), 5.

23. "Assises Québec-municipalités..." " 6.

24. Lucien Bélanger, "Les municipalités à l'ère de la tarification.", Municipalité, (février 1990), 16. 


\begin{tabular}{|c|c|c|c|c|c|}
\hline \multirow[b]{2}{*}{ Municipalités } & \multicolumn{5}{|c|}{$\begin{array}{c}\text { Tableau } 13 \\
\text { Changements }(+/-) \text { des subventions du ministère des Affaires culturelles } \\
\text { accordées aux bibliothèques municipales } \\
\text { (Année de base : 1984) }\end{array}$} \\
\hline & Poste & 1985 & 1986 & 1987 & 1988 \\
\hline 10 & Subventions & $-34.2 \%$ & $-29.0 \%$ & $0.0 \%$ & $+26.9 \%$ \\
\hline 11 & Subventions & $-17.6 \%$ & $-29.0 \%$ & $0.0 \%$ & $+27.0 \%$ \\
\hline 12 & Subventions & $+\quad 1.2 \%$ & $-29.0 \%$ & $0.0 \%$ & $+26.9 \%$ \\
\hline 13 & Subventions & $+\quad 8.7 \%$ & $-29.0 \%$ & $+\quad 27.5 \%$ & $-\quad 0.5 \%$ \\
\hline 15 & Subventions & $+13.7 \%$ & $-29.0 \%$ & $0.0 \%$ & $+27.0 \%$ \\
\hline 16 & Subventions & $+23.9 \%$ & $-29.0 \%$ & $0.0 \%$ & $+27.0 \%$ \\
\hline 17 & Subventions & $+70.7 \%$ & $-28.4 \%$ & $0.0 \%$ & $+27.0 \%$ \\
\hline 18 & Subventions & - $\quad 6.3 \%$ & $-29.0 \%$ & $0.0 \%$ & $+19.5 \%$ \\
\hline 19 & Subventions & $+45.3 \%$ & $-39.8 \%$ & $-\quad 2.3 \%$ & $+50.1 \%$ \\
\hline 20 & Subventions & $+\quad 4.2 \%$ & $-29.0 \%$ & $0.0 \%$ & $+27.0 \%$ \\
\hline 24 & Subventions & $+27.9 \%$ & $-29.0 \%$ & $0.0 \%$ & $+27.0 \%$ \\
\hline 25 & Subventions & $+157.5 \%$ & $-35.9 \%$ & $+156.7 \%$ & $-50.6 \%$ \\
\hline 27 & Subventions & $-\quad 2.2 \%$ & $-29.0 \%$ & $0.0 \%$ & $+27.0 \%$ \\
\hline 28 & Subventions & $-\quad 7.1 \%$ & $-29.0 \%$ & $0.0 \%$ & $+27.0 \%$ \\
\hline 29 & Subventions & $+18.0 \%$ & $-29.0 \%$ & $0.0 \%$ & $+26.9 \%$ \\
\hline 31 & Subventions & $+12.5 \%$ & $-29.0 \%$ & $0.0 \%$ & $+27.0 \%$ \\
\hline 34 & Subventions & $-14.5 \%$ & $-29.0 \%$ & $0.0 \%$ & $+27.0 \%$ \\
\hline 35 & Subventions & $+59.9 \%$ & $-44.4 \%$ & $0.0 \%$ & $+27.0 \%$ \\
\hline 38 & Subventions & - $^{*}$ & - & - & - . \\
\hline 40 & Subventions & $-8.3 \%$ & $-29.0 \%$ & $0.0 \%$ & $+27.0 \%$ \\
\hline 41 & Subventions & $-\quad 1.0 \%$ & $-29.0 \%$ & $0.0 \%$ & $+26.9 \%$ \\
\hline 42 & Subventions & $+\quad 5.3 \%$ & $-34.8 \%$ & $0.9 \%$ & $+27.0 \%$ \\
\hline
\end{tabular}

Parmi les autres facteurs susceptibles d'influencer favorablement les décisions des élus, il y a bien sûr les demandes des citoyens. Encore fautil que ces demandes soient exprimées manifestement, sans ambages, et de façon soutenue. Un moyen efficace et souvent utilisé par les citoyens pour exprimer leurs demandes consiste à se regrouper. Ainsi dans la communauté on retrouve de nombreux Club..., Association..., Regroupement..., porte-parole et gardiens d'une plus grande "qualité de vie" dans leur milieu. Leur influence n'est peut-être pas toujours immédiate, mais elle est présente. La bibliothèque, par contre, ne peut compter sur un tel appui ${ }^{25}$. Bien que souvent $30 \%$ ou même $50 \%$ des citoyens de la communauté utilisent ses services, une visite à la bibliothèque n'est que très rarement une action de groupe. Tenant compte des résultats de la présente étude, l'administration municipale ne semble pas disposée à faciliter la formation d'un tel regroupement, puisque le facteur "Organiser, sur une base annuelle, une rencontre avec les citoyens pour discuter des services offerts par la bibliothèque" se retrouve au tableau 2 mais non au tableau 1.

Le commentaire de l'élu, celui du responsable de bibliothèque, et le commentaire du directeur général, déjà cité, illustrent nos propos au sujet de l'influence de la hiérarchie administrative dans la prise de décision des élus. De plus, sur le plan hiérarchique, dans la plupart des cas, le dossier " bibliothèque " est analysé, examiné, évalué attentivement par au moins deux paliers administratifs avant d'atteindre le conseil. Un tel cheminement n'a rien de répréhensible en soi, mais si à ce cheminement par paliers on ajoute des différences marquées de perception au niveau de l'essence même du service, un dossier amenuisé risque de parvenir au conseil.

Il va sans dire que les deux derniers facteurs portant sur la compétition et la visibilité sont plus difficiles à observer et à mesurer. Cependant, si l'idée stéréotypée de «la petite fille de la bibliothèque " et celle "des livresdécrépits " nous viennent à l'esprit, le profil des répondants de cette enquête 26 montre que $69 \%$ des direc-

25. "Bibliothèques publiques: le gouvernement ne dépose pas son projet de loin; Urba, vol. 11, no 6 (juillet-août 1990), 8. 
teurs de bibliothèques sont des femmes et que $54 \%$ de ce groupe a moins de 40 ans. Par contre, $85 \%$ des élus sont des hommes et $35 \%$ d'entre eux ont plus de 50 ans. Un phénomène semblable se produit pour les directeurs généraux et les directeưrs hiérarchiques bien que ces deux groupes soient en moyenne légèrement plus jeunes.

Ces résultats $n^{\prime}$ ont sans doute aucun lien avec les différences de perception observées dans cette étude, sauf, peutêtre au niveau des communications entre les groupes. En plus des commentaires déjà présentés, où les répondants expriment certaines difficultés, il faut rappeler une différence significative de perception entre "discuter de la bibliothèque avec le responsable en dehors des réunions du conseil" et "visiter la bibliothèque régulièrement " (tableau 3). De plus, il convient de rappeler que seulement $38 \%$ des directeurs de bibliothèques sont $d$ 'avis que la structure municipale permet aux élus municipaux de discuter directement avec eux (communications descendantes), tandis que plus de $75 \%$ de chacun des autres groupes partagent cet avis. D'autre part, seulement $31 \%$ des directeurs de bibliothèques estiment que cette structure leur permet de discuter directement avec les élus (communications ascendantes) ${ }^{27}$.

Alors que peu de facteurs externes et internes semblent favoriser la bibliothèque, il est possible, à notre avis, qu'un problème de communications influence. les perceptions des différents groupes concernés et que le support financier consenti à la bibliothèque soit le résultat de ces perceptions. D'ailleurs, Maud Lefebvre-Roux, dans son évaluation critique des programmes de subventions du ministère des Affaires culturelles, soutient:

Il est essentiel d'informer nos élus municipaux du peu de garantie qu'offrent ces alléchants program- mes de subvention. Notre responsabilité est d'impliquer les décideurs politiques municipaux dans un véritable développement des bibliothèques publiques québécoises et ce, dans une perspective globale...

Il est de notre ressort de développer l'argumentation qui saura influencer nos élus municipaux à investir dans le développement de LEUR bibliothèque municipale ${ }^{28}$.

26. Diane Mittermeyer, "La bibliothèque publique et le milieu... ", 147.

27. Ibid., 159.

28. Maud Lefebvre-Roux, "Chers(ères) collègues $" . . ., 5$.

Note: En se référant au premier article, nos lecteurs voudront bien corriger deux erreurs qui se sont glissées lors de sa publication. II faudrait lire dans le titre du résumé anglais: "... Part I: The Opinion of Municipal Governments " et à la p. 149, sous le titre "Les résultats", lire "...les choix possibles étaient: ESSENTIEL... " et non "essentiellement". Nous nous en excusons auprès de I'auteure.

\section{The Annotated Copyright Act 1991}

\section{par Normand Tamaro}

V oici un guide de travail vraiment complet présentant un portrait à jour et cohérent du droit d'auteur canadien dans un contexte moderne.

Vous y trouverez le texte intégral de la Loi sur le droit d'auteur et ses règlements, de même que le texte de la Loi québécoise sur le statut professionnel des artistes des arts visuels, des métiers d'arts et de la littérature et sur les contrats avec les diffuseurs.

L'auteur fournit des commentaires détaillés faisant ressortir les principes généraux du droit d'auteur au Canada, en plus d'extraits tirés intégralement des arrêts. L'ouvrage inclut enfin un index général complet et méthodique.

$$
\text { 0-459-35051-*/ février 1991/Couverture rigide }
$$

Approximativement 576 pages/ Prix non encore fixé

Disponible pour examen gratuit de 30 jours

Thomson Professional Publishing Cạnada

\section{D Carswell / Richard De Boo Publishers-Éditeur}

Veuillez faire parvenir vos commandes au:

Corporate Plaza, 2075 Kennedy Road, Scarborough, Onțario, M1T 3V4

Télécopieur: (416) 298-5094 (24 heures)

SANS FRAIS: 1-800-387-5164 (de 8 b00 à 20h00, heure normale de l'est).

A Toronto: (416) 609-3800

Les prix peuvent être modifiés sans préavis. 